ARTICLE

DOI: $10.1038 / \mathrm{s} 41467-017-02585-y$

\title{
Interaction of suppressor of cytokine signalling 3 with cavin-1 links SOCS3 function and cavin-1 stability
}

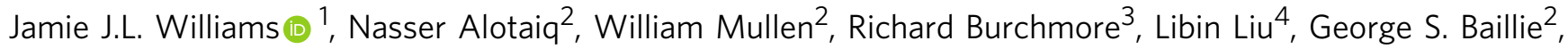
Fred Schaper ${ }^{5}$, Paul F. Pilch ${ }^{4} \&$ Timothy M. Palmer (i) ${ }^{1}$

Effective suppression of JAK-STAT signalling by the inducible inhibitor "suppressor of cytokine signalling 3" (SOCS3) is essential for limiting signalling from cytokine receptors. Here we show that cavin-1, a component of caveolae, is a functionally significant SOCS3interacting protein. Biochemical and confocal imaging demonstrate that SOCS3 localisation to the plasma membrane requires cavin-1. SOCS3 is also critical for cavin-1 stabilisation, such that deletion of SOCS3 reduces the expression of cavin- 1 and caveolin-1 proteins, thereby reducing caveola abundance in endothelial cells. Moreover, the interaction of cavin-1 and SOCS3 is essential for SOCS3 function, as loss of cavin-1 enhances cytokine-stimulated STAT3 phosphorylation and abolishes SOCS3-dependent inhibition of IL-6 signalling by cyclic AMP. Together, these findings reveal a new functionally important mechanism linking SOCS3-mediated inhibition of cytokine signalling to localisation at the plasma membrane via interaction with and stabilisation of cavin-1.

\footnotetext{
${ }^{1}$ School of Pharmacy and Medical Sciences, University of Bradford, Bradford BD7 1DP, UK. ${ }^{2}$ Institute of Cardiovascular and Medical Sciences, University of Glasgow, Glasgow G12 8QQ, UK. ${ }^{3}$ Polyomics Facility, University of Glasgow, Glasgow G12 8QQ, UK. ${ }^{4}$ Departments of Biochemistry and Medicine, Boston University School of Medicine, Boston MA 02118, USA. ${ }^{5}$ Department of Systems Biology, Institute for Biology, Otto-von-Guericke-University Magdeburg 39106 Magdeburg, Germany. Correspondence and requests for materials should be addressed to J.J.L.W. (email: j.j.l.williams@bradford.ac.uk) or to T.M.P. (email: T.Palmer1@bradford.ac.uk)
} 
C ytokines control many important biological responses, including haematopoiesis, T-cell differentiation and expansion, and inflammatory status ${ }^{1,2}$. Multiple temporally distinct inhibitory mechanisms operate to ensure signalling responses downstream of activated cytokine receptors are transient in nature. Therefore, sustained pathway activation perpetuates chronic inflammatory conditions such as rheumatoid arthritis and colitis, haematological malignancies such as polycythemia vera, and also solid tumour development ${ }^{3-5}$.

Several cytokine receptors, including gp130 (the signal transducing component of the interleukin-6 (IL-6) signalling complex), activate receptor-associated Janus kinases (JAKs) which then trigger receptor engagement with proteins such as signal transducer and activators of transcription (STATs), particularly STAT3. Phosphorylated STATs can then dimerise and translocate to the nucleus, where they function as transcription factors by binding to specific promoter elements and recruiting transcriptional co-activators ${ }^{1,2}$.

"Suppressors of cytokine signalling" (SOCS) proteins comprise a family of eight related members (cytokine-inducible $\mathrm{SH} 2$ containing protein (CIS), SOCS1-7) identified initially by their role as cytokine-inducible negative feedback inhibitors of signal propagation from specific cytokine receptors ${ }^{6}$. SOCS3 is recruited to activated cytokine receptors following the formation of a SOCS3 interaction motif upon phosphorylation of key Tyr residues by cytokine-activated JAKs. SOCS3 terminates signalling from gp130 by binding via a central SH2 domain to PTyr759, allowing it to interact with and inhibit adjacent receptor-bound JAKs via its kinase inhibitory region (KIR) thereby preventing the recruitment and tyrosine phosphorylation of STATs ${ }^{7}$. The Cterminal SOCS box domain directs $\mathrm{SH} 2$ domain-bound interacting proteins for ubiquitylation due to its ability to bind elongin $\mathrm{B}$ and C, Cullin family member Cul5, and RING (Really Interesting New Gene) finger protein $\mathrm{Rbx}^{7}$. Following SOCS3dependent ubiquitylation, targets such as FAK1 can be degraded either by the proteasome $e^{8,9}$ or, in the case of the granulocyte colony-stimulating factor receptor (G-CSFR), by trafficking into lysosomal compartments following internalisation ${ }^{10}$. However, despite advances in our molecular understanding of how SOCS3 interacts with cytokine receptors and JAKs, the extent to which other cellular proteins regulate SOCS3 function is unclear. Recently, CUE domain-containing 2 (CUEDC2) was identified as a novel SOCS3-interacting protein that could enhance its interaction with elongin $C^{11}$. Such observations raise the possibility that additional protein interactors may be required to maximise the ability of SOCS3 to regulate signalling.

Cavin-1 (alternatively known as polymerase I and transcript release factor (PTRF)) is an abundant component of caveolae, which function as specialised lipid raft microdomains within the plasma membrane. Caveolae were first identified by electron microscopy as $50-100 \mathrm{~nm}$ flask-shaped plasma membrane invaginations $^{12}$ and are now known to play critical roles in controlling endocytosis, sphingolipid metabolism, and compartmentalisation of signalling pathways ${ }^{13}$. Cavin-1, which is one of a family of four related proteins (cavins 1 to 4), is recruited by one or more "caveolin" proteins (caveolins 1 to 3 ) to the plasma membrane during the latter stages of caveola biogenesis, and is thought to be essential for caveola formation by stabilising caveolin proteins at the plasma membrane ${ }^{14}$.

While some studies have demonstrated localisation of cytokine receptors and JAKs in lipid raft microdomains ${ }^{15-18}$, little is known about the impact of caveolin expression/function on JAK-STAT signalling and no studies have specifically examined a role for cavins. In this study, we identify a novel interaction between SOCS3 and cavin-1. This interaction is not only required for optimal SOCS3-mediated inhibition of IL-6-mediated
JAK-STAT signalling but also for effective stabilisation of cavin-1 and hence caveolin-1. Therefore, our findings define a new relationship between SOCS3 and cavin-1 in which each partner plays previously unappreciated roles in maintaining effective inhibition of JAK-STAT signalling (cavin-1), cavin-1 expression, and caveola stability (SOCS3).

\section{Results}

Cavin-1 as a SOCS3-regulated ubiquitylated protein. As well as inhibiting cytokine receptor signalling by inhibiting the Tyr kinase activity of receptor-bound $\mathrm{JAKs}^{19}$, SOCS3 can also control the stability of $\mathrm{SH} 2$ domain-bound proteins as part of an elongin/ cullin/SOCS3 $\left(\mathrm{ECS}^{\mathrm{SOCS} 3}\right)$ E3 ubiquitin ligase complex ${ }^{6,7,20}$. While several ubiquitinated substrates of SOCS3 are known, the full spectrum has yet to be identified. Thus, we pursued an experimental approach to elaborate on SOCS3 function by investigating SOCS3-regulated proteins. Since there is no consensus sequence for ubiquitylation, we used a stable isotopic labelling of amino acids in cell culture (SILAC)/mass spectrometry approach to compare ubiquitinomes from wild-type SOCS3 ${ }^{+/+}$(WT) and SOCS3 ${ }^{-/-}$murine embryonic fibroblast (MEF) cell lines expressing equivalent levels of a tandem affinity purification-compatible tagged ubiquitin transgene following SOCS3 induction (Supplementary Fig. 1). Using this approach, ubiquitylated proteins regulated by SOCS3 would be predicted to be enriched in WT but not SOCS3 ${ }^{-/-}$MEFs.

A ubiquitin transgene containing a tandem hexahistidine and biotin tag (HB-Ub) was used to allow two-step tandem affinity purification of the ubiquitinome via sequential Ni-NTA and streptavidin affinity chromatography under fully denaturing conditions necessary to inactivate deubiquitinases and prevent co-purification of non-covalently interacting ubiquitin-binding proteins $^{21,22}$. Stable HB-Ub-expressing WT and SOCS3 ${ }^{-/-}$MEFs were generated via retrovirus-mediated gene transfer and assessed by immunoblotting for equivalent expression levels of the HB-Ub transgene. SOCS3 was induced by elevation of intracellular cyclic adenosine monophosphate (cAMP) levels with forskolin $(50 \mu \mathrm{M})$, a direct activator of adenylyl cyclase. To increase the probability of detecting proteins whose ubiquitylation was SOCS3 dependent, protein tyrosine phosphatases were inhibited using a combination of sodium orthovanadate and $\mathrm{H}_{2} \mathrm{O}_{2}$ to preserve the PTyr status of potential substrates and maximise interaction with the SOCS3 SH2 domain ${ }^{23}$, while the ubiquitinome was preserved using proteasome inhibitor MG132. Tandem affinity-purified ubiquitinomes from WT and SOCS3 ${ }^{-/-}$MEFs were then analysed using an Orbitrap Velos FTMS and data processed using the MaxQuant computational platform $^{24}(170)$. Under these conditions, MaxQuant detected cavin-1 (O54724) with a $\log _{2}$ (normalised $\mathrm{H} \mathrm{L}^{-1}$ ) $=1.37$ ( 5 unique peptides, count ratio of 6 ). This suggested that cavin-1 was a ubiquitylated protein specifically depleted in $\mathrm{SOCS}^{-/-}$MEFs.

SOCS3 enhances cavin-1 stability. Our proteomics screen suggested that SOCS3 regulates cavin-1 stability. To examine this, we compared the ability of increasing levels of SOCS3 to trigger the proteasomal degradation of co-transfected cavin-1 and FAK1, a previously characterised substrate of $\mathrm{ECS}^{\mathrm{SOCS} 38,9}$. Consistent with previous work, increased SOCS3 expression triggered a decrease in FAK1 levels that could be rescued by inclusion of proteasome inhibitor MG132. In contrast, levels of cavin-1 were not altered even at the highest level of SOCS3 expression and were not increased by proteasome inhibitor MG132 (Fig. 1a). To determine whether SOCS3 could regulate levels of endogenous cavin-1, we assessed the effects of SOCS3 deletion on cavin-1 expression levels in MEFs. Immunoblotting of whole-cell extracts 
a

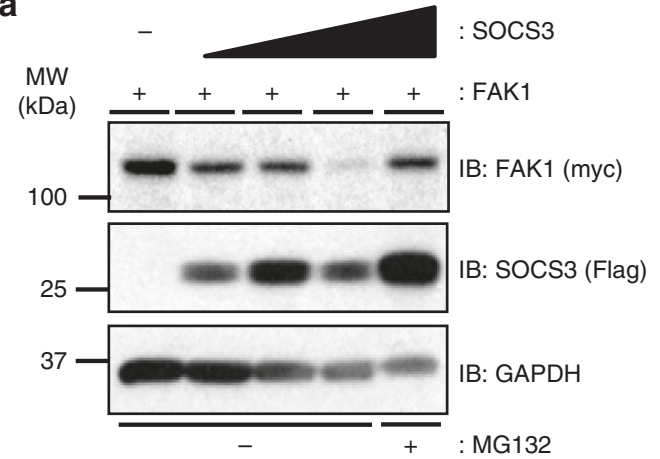

b

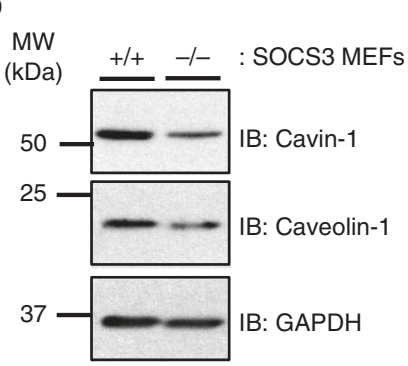

C

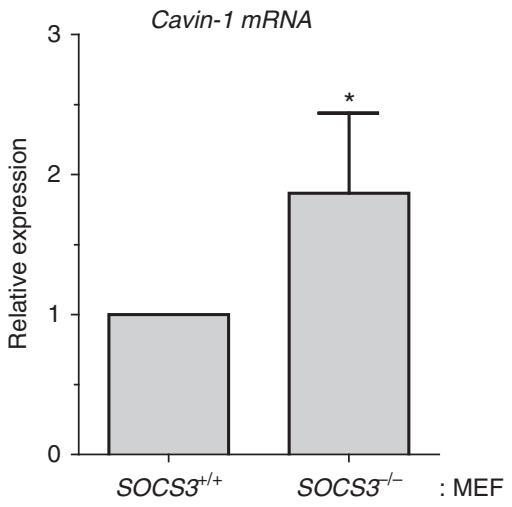

d
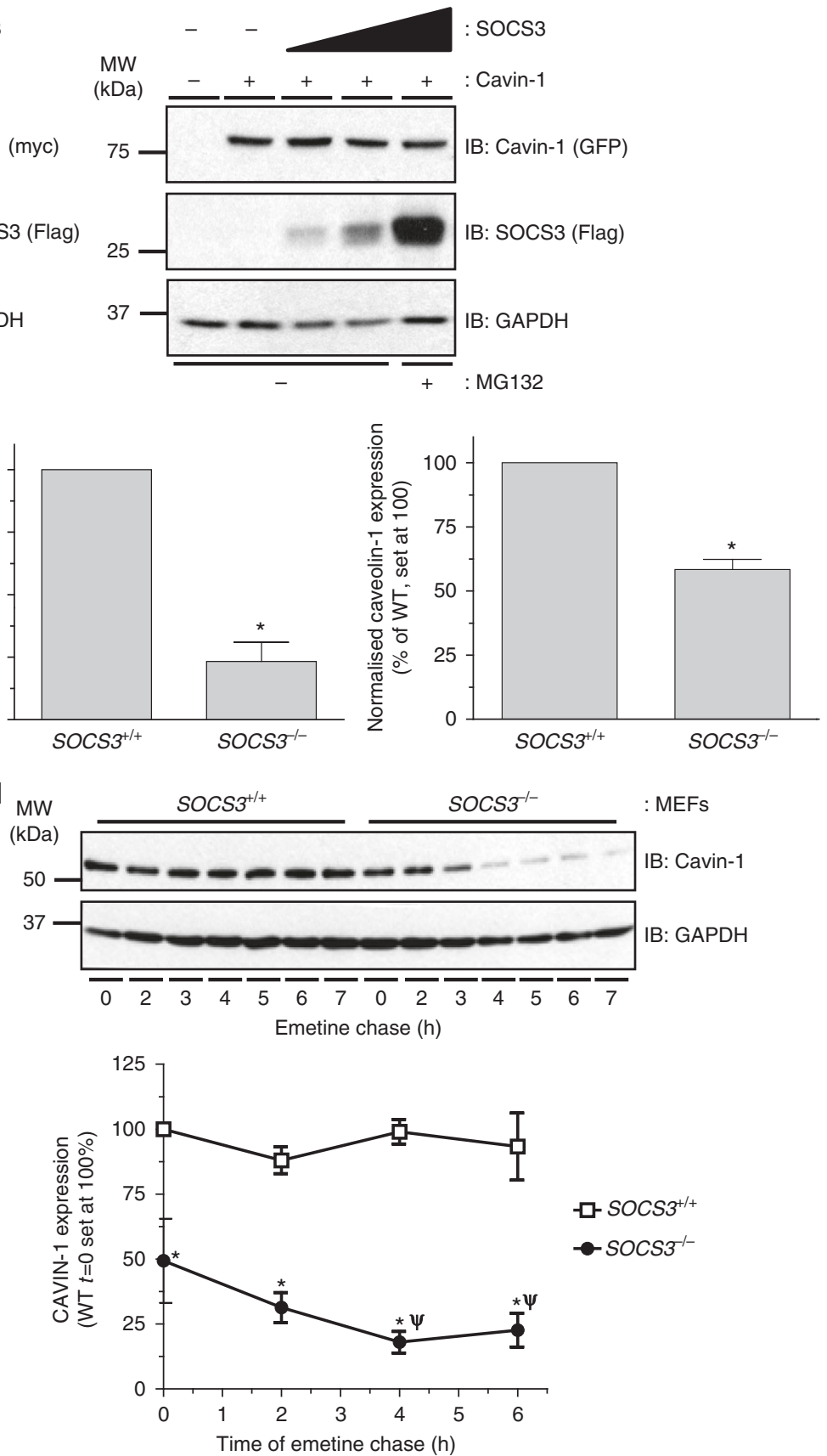

Fig. 1 Cavin-1 stability is enhanced in the presence of SOCS3. a HEK293 cells co-transfected with fixed amounts of either myc-tagged FAK1 or GFP-cavin-1 expression constructs and increasing levels of Flag-SOCS3 were treated with or without proteasome inhibitor MG132 (6 $\mu \mathrm{M})$ as indicated. Detergentsoluble whole-cell lysates were analysed by SDS-PAGE and immunoblotting. b Detergent-soluble whole-cell lysates prepared from SOCS3 ${ }^{+/+}(+/+)$and SOCS3 $^{-/-}(-/-)$MEFs equalised for protein content were analysed by SDS-PAGE and immunoblotting. c Quantitative real-time PCR of cavin-1 mRNA levels in WT $\left(\mathrm{SOCS}^{+/+}\right)$and SOCS3 ${ }^{-/-}$MEFs. Data are presented as mean \pm standard error for $N=3$ experiments. d Upper: Protein-equalised soluble cell extracts from $\mathrm{SOCS}^{+/+}$and SOCS3 ${ }^{-/-}$MEFs chased for the indicated times in serum-free medium with protein synthesis inhibitor emetine $(100 \mu \mathrm{M})$ were analysed by SDS-PAGE and immunoblotting with the indicated antibodies. Lower: Quantitation of cavin- 1 levels in SOCS3 ${ }^{+/+}$and SOCS3 ${ }^{-/-}$MEFs is also shown. Results are presented as mean values \pm standard error for $N=3$ experiments. ${ }^{\star} P<0.05$ vs. corresponding treatment in SOCS3 ${ }^{+/+} \mathrm{MEFs},{ }^{\psi} P<0.05$ vs. $t=0$

revealed that levels of cavin-1 protein were significantly reduced in $\mathrm{SOCS3}^{-/-}$MEFs versus WT cells. This reduction occurred under conditions in which cavin-1 mRNA levels were significantly increased in SOCS3 ${ }^{-/-}$MEFs versus WT cells (Fig. 1b, c). The decrease in cavin-1 protein was paralleled by a similar decrease in caveolin-1 expression levels (Fig. 1b), which is consistent with previous studies showing that loss of cavin-1 triggers reductions in all three caveolin isoforms ${ }^{14}$. We then measured the half-lives of cavin-1 protein in WT and $S O C S 3^{-/-}$MEFs by monitoring time-dependent changes in cavin-1 expression in whole-cell extracts following inhibition of protein synthesis with emetine ${ }^{25}$. For these experiments, cells were also pre-treated with forskolin (Fsk) to elevate cAMP levels and increase SOCS3 expression in WT $\mathrm{MEFs}^{26,27}$ Direct comparison of cavin-1 
a

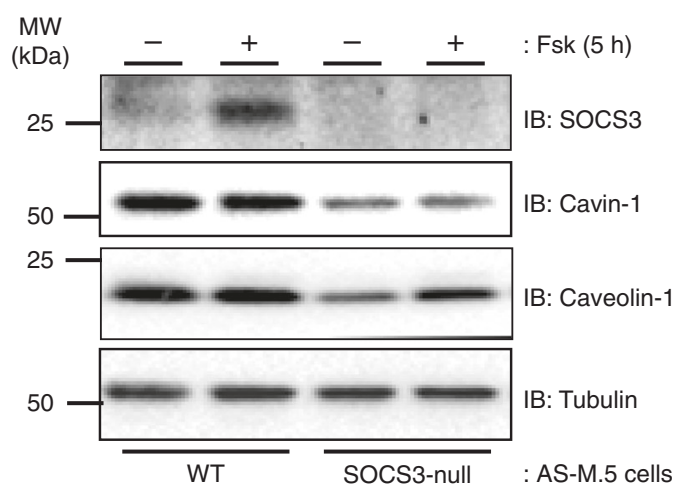

b WT

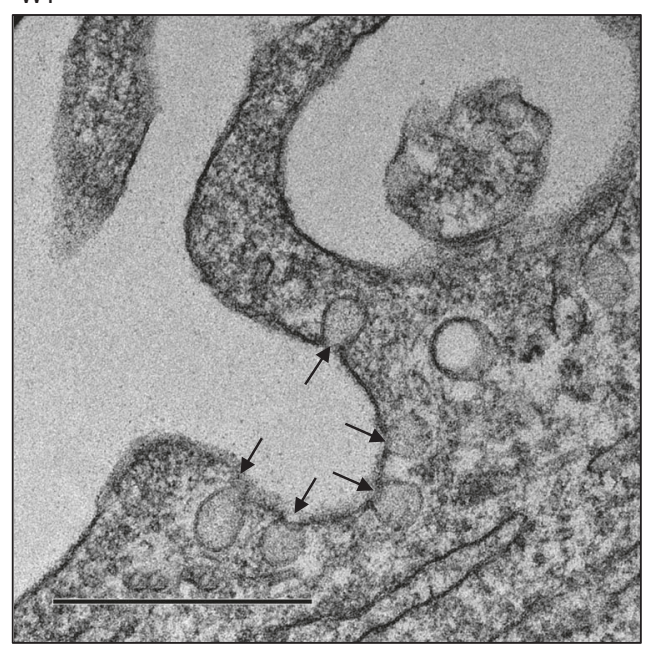

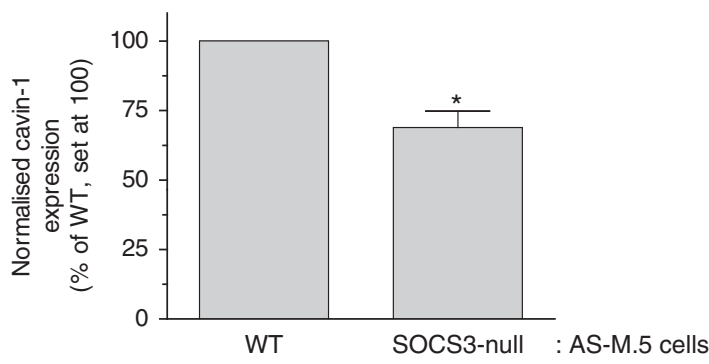

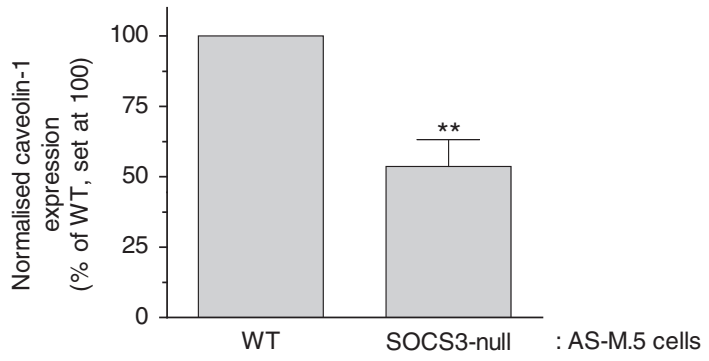

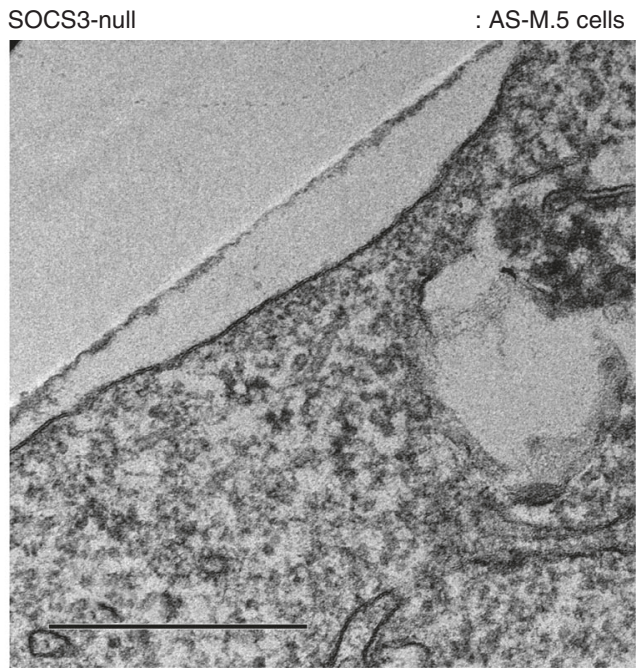

C

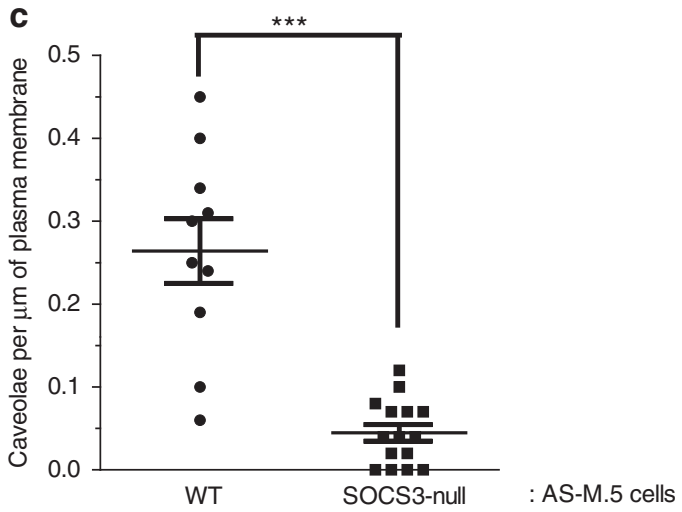

Fig. 2 Effect of SOCS3 deletion on caveola abundance in endothelial cells. a Upper: Detergent-soluble whole-cell lysates from WT and SOCS3-null AS-M.5 human angiosarcoma-derived ECs treated with either vehicle or $50 \mu \mathrm{M}$ Fsk for $5 \mathrm{~h}$ were equalised for protein content for SDS-PAGE for immunoblotting with the indicated antibodies. Lower: Quantitation of cavin-1 and caveolin-1 protein levels in unstimulated AS-M.5 cells is presented as mean \pm standard error for $N=3$ experiments. ${ }^{\star} P<0.05,{ }^{\star \star} P<0.01$ vs. WT cells. b Transmitting electron microscopy (TEM) was performed on WT and SOCS3-null AS-M.5 cells as indicated. Cell surface caveolae (indicated by the arrows) were readily detectable in WT cells (left panel). In contrast, plasma membranes from SOCS3-null cells were flat and caveolae density was significantly reduced compared to WT cells (right panel). Scale bar $=0.5 \mu \mathrm{m}$. c Quantitation of caveola density (number of caveolae per $\mu \mathrm{m}$ of plasma membrane) in WT and SOCS3-null AS-M.5 cells. ${ }^{\star \star \star} P<0.0001$ vs. WT cells 
stability in WT versus SOCS3 ${ }^{-/}$MEFs demonstrated that the absence of SOCS3 significantly reduced the half-life from $>8 \mathrm{~h}$ (WT MEFs) to $2 \mathrm{~h}$ (SOCS3 $3^{-1}$ MEFs: Fig. 1d). Thus, in contrast to the well-defined role of SOCS3 in destabilising target proteins by targeting them for ubiquitylation and proteasomal degradation, the presence of SOCS3 stabilised cavin-1.

Effect of SOCS3 deletion on caveola abundance. Our data thus far suggested that SOCS3 was an important regulator of caveolin1 abundance via stabilisation of cavin-1. Homozygous deletion of the cavin-1 gene in mice results in marked reductions in the expression of all caveolin isoforms and a lack of detectable caveolae in multiple cell types, including endothelial cells (ECs) in which caveolae are especially abundant ${ }^{14}$.

To examine the impact of SOCS3 on caveola abundance, clustered regularly interspaced short palindromic repeats (CRISPR)/Cas9 technology was used to generate SOCS3-null AS-M.5 human angiosarcoma-derived immortalised $\mathrm{ECs}^{28}$. Treatment of WT AS-M.5 cells with cAMP-elevating agent Fsk was able to promote SOCS3 induction similar to that observed in MEFs and primary EC lines as previously reported ${ }^{26,27}$ However, this effect was lost in SOCS3-null AS-M.5 cells, while Nur77, a well-characterised cAMP-inducible gene product ${ }^{29}$, was detectable in both WT and SOCS3-null AS-M.5 cells following Fsk treatment (Fig. 2a, Supplementary Fig. 2). Similar to MEFs (Fig. 1b), SOCS3 deletion significantly reduced cavin-1 and caveolin-1 protein levels in AS-M.5 whole-cell extracts (Fig. 2a), demonstrating that this effect is independent of the cell system being investigated.

We then used transmission electron microscopy (TEM) to assess any consequences of the observed changes in cavin- 1 and caveolin-1 expression on the abundance of cell surface caveolae. Caveolae were readily detectable in WT AS-M.5 cells as plasma membrane-localised flask-shaped invaginations ranging from 50 to $100 \mathrm{~nm}$ in diameter (Fig. 2b). In contrast, these were barely detectable in SOCS3-null cells (Fig. 2b, c). Therefore, significant reductions in cavin-1 and caveolin-1 protein levels triggered by the loss of SOCS3 in endothelial cells are translated into significantly reduced numbers of cell surface caveolae.

Cavin-1 interacts with SOCS3 via a SH2 domain PEST sequence. To assess whether SOCS3 could directly interact with cavin-1, co-immunoprecipitation (co-IP) experiments were performed in lysates isolated from transfected HEK293 cells transiently expressing Flag-SOCS3 and myc-cavin-1. These experiments demonstrated that myc-cavin-1 was present in antiFlag antibody immunoprecipitates only when co-expressed with Flag-SOCS3, indicating the two proteins formed a complex (Fig. 3a). Similar results were obtained using Flag-cavin-1 and HA-SOCS3 (Supplementary Fig. 3), indicating that the effect was independent of the combination of tags used. Analysis of lysates and unbound samples from the experiments demonstrated that under conditions in which SOCS3 could be fully precipitated from lysates, a proportion of cavin-1 remained unbound, suggesting that not all available cavin-1 could interact with SOCS3 under these condition (Supplementary Fig. 4). To assess the interaction of endogenously expressed SOCS3 and cavin-1, WT and SOCS3-null AS-M.5 cells were stimulated with Fsk prior to IP of cavin-1 and analysis by immunoblotting. These experiments demonstrated that immunoreactive SOCS3 was specifically enriched in cavin-1 IPs from WT AS-M.5 cells (Fig. 3b), consistent with the co-IP data from experiments using transfected cells (Fig. 3a).

To identify the regions within SOCS3 that are important for SOCS3/cavin-1 interaction, we initially utilised a panel of Flag- tagged $\mathrm{N}$ - and C-terminal SOCS3 truncation mutants $\mathrm{s}^{30,31}$ for their ability to co-IP green fluorescent protein (GFP)-tagged cavin- ${ }^{32}$ as compared to WT SOCS3. Interestingly, all of the truncation mutants tested were able to co-IP GFP-cavin- 1 to the same extent as full-length WT SOCS3 (Fig. 3c), suggesting that a region within the $\mathrm{SH} 2$ domain present in each of the mutants was necessary for SOCS3 binding to cavin-1. To test this, we expressed full-length SOCS3 (residues 1-225) and the region of the SOCS3 SH2 domain (residues 46-142, termed SOCS3 $\Delta \mathrm{SH} 2$ ) required for cavin-1 binding (Fig. 3d) as GFP-tagged fusion proteins and compared their ability to co-IP myc-tagged cavin-1 in transfected HEK293 cells. As a negative control, we used a GFP fusion protein containing residues $177-225$ of the SOCS box that we identified as dispensable for cavin-1 interaction (Fig. 3c). Similar to WT SOCS3-GFP, SOCS3 $\triangle$ SH2-GFP was able to co-IP myc-tagged cavin-1 above the non-specific levels observed with the SOCS3 SOCS box-GFP fusion and cavin-1 alone, albeit not to the same extent as WT SOCS3-GFP (Fig. 3d, lane 2 versus lane 8). Therefore, these data showed that residues 46-142 within the SOCS3 SH2 domain were both necessary and sufficient for SOCS3 interaction with cavin-1.

As many SOCS3 binding partners, including gp 130, CD33 and FAK1, must be Tyr phosphorylated in order to interact with SOCS3, we pursued three experimental approaches to examine whether or not the PTyr-binding pocket within the SOCS3 SH2 domain was required for interaction with cavin-1. First, we treated transfected HEK293 cells with protein Tyr phosphatase inhibitor sodium orthovanadate in the presence or absence of hydrogen peroxide ${ }^{23}$. These experiments demonstrated that the isolation of GFP-cavin-1 in anti-Flag (SOCS3) immunoprecipitates was not altered by increases in global Tyr phosphorylation levels (Fig. 4a), suggesting that cavin-1 formed a complex with SOCS3 via a mechanism that did not require prior Tyr phosphorylation. Secondly, we tested R71K-mutated SOCS3, in which the conserved PTyr binding site within the SOCS3 SH2 domain is disrupted ${ }^{30,31}$, for its ability to form a complex with cavin-1. Co-IP assays revealed that a R71K-mutated SOCS3 bound cavin-1 equivalently to WT SOCS3 (Fig. 4b), again supporting the concept that cavin-1 interacted with the SOCS3 $\mathrm{SH} 2$ domain in a manner independent of its capacity to bind Tyrphosphorylated ligands. Finally, N-terminally biotinylated peptides encompassing the Tyr759 motif of gp130 in phosphorylated (PTyr759 peptide) and non-phosphorylated (Tyr759 peptide) forms were used as bait to test the effect of cavin-1 co-expression on the ability of SOCS3 to be precipitated in peptide pull-down assays. As reported by others ${ }^{30}$, SOCS3 specifically associated with the PTyr759 peptide under these conditions. Using a maximally effective concentration of peptide $(100 \mathrm{nM})$, coexpression with cavin-1 did not reduce the ability of SOCS3 to precipitate with PTyr759 peptide (Fig. 4c). Taken together, these data demonstrated that cavin-1 interacted with the SOCS3 SH2 domain at a location distinct from the well-defined PTyr-binding pocket.

The region of the SOCS3 SH2 domain identified in the studies above consists of two structurally distinct components. Firstly, residues 46-127 comprise of $\beta$-sheet and $\alpha$-helical regions that form part of the PTyr-binding pocket common to all SH2 domains. Secondly, residues 128-142 form part of an unstructured PEST sequence insert that links the SH2 domain helix B with BG loop and $\beta G$ strand motifs (residues 166-185) ${ }^{33}$. PEST motifs are unstructured hypermobile regions that have roles in multiple cellular processes by controlling protein-protein interactions and protein turnover ${ }^{34,35}$. We noted the presence of a PEST sequence within the classic $\mathrm{SH} 2$ domain structure is also displayed by CIS but none of the other SOCS family protein ${ }^{33}$. Having excluded the PTyr-binding functionality for $\mathrm{SH} 2$ domain 
a

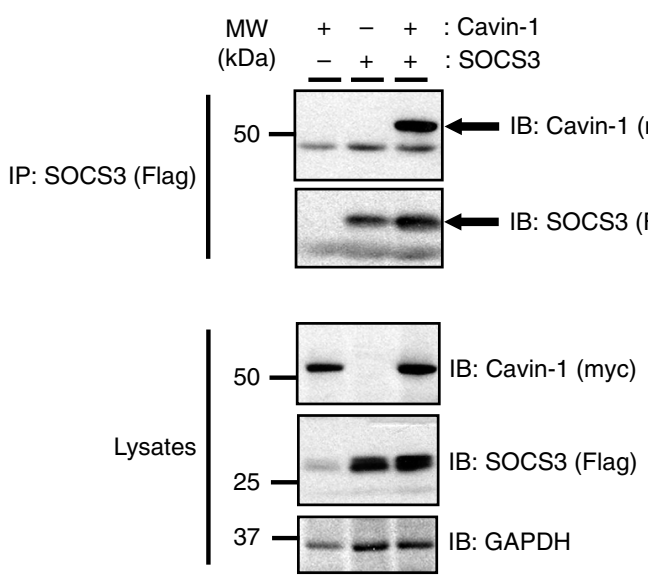

d
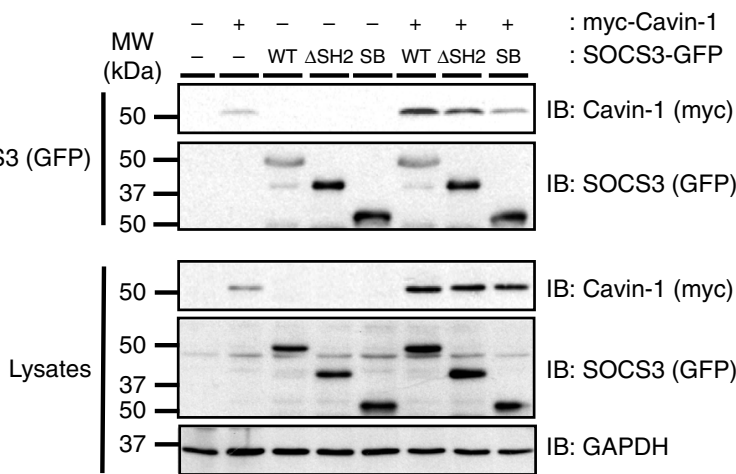

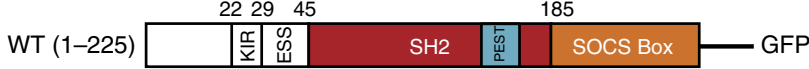

$\Delta \mathrm{SH} 2$ domain (46-142)

$\mathrm{SH} 2$

SOCS box (177-225)

C

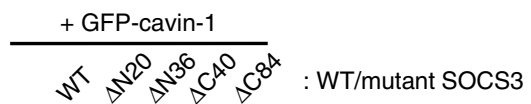

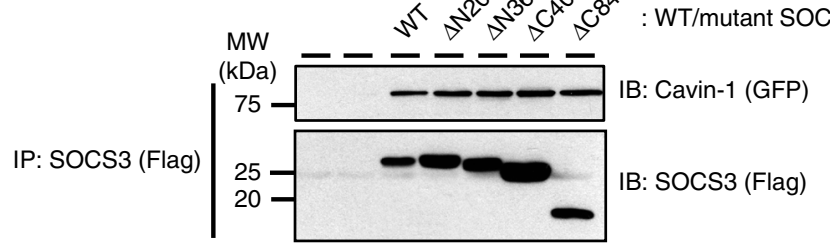

b
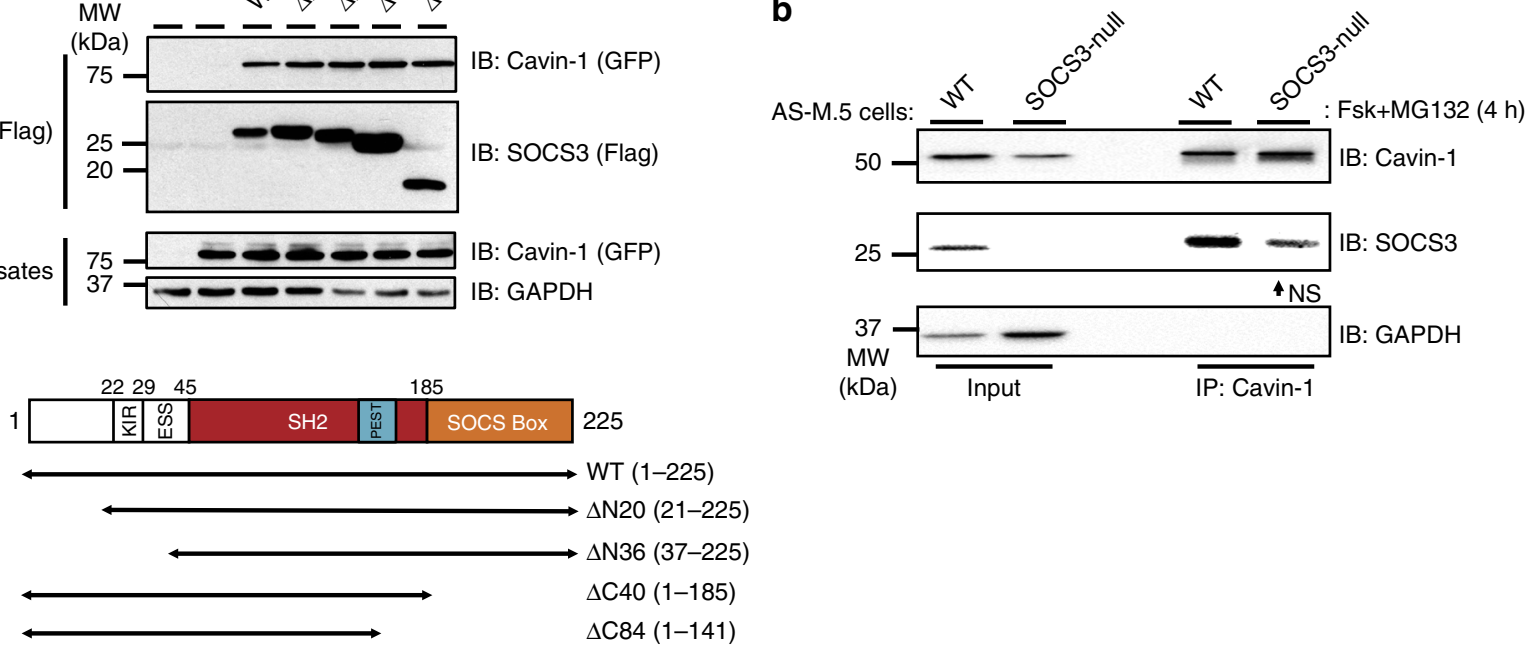
\begin{tabular}{l|lll} 
Lysates & $75 \rightarrow$ IB: Cavin-1 (GFP) \\
37 & IB: GAPDH
\end{tabular} $\Delta \mathrm{C} 84(1-141)$

Fig. 3 A region within the SOCS3 SH2 domain is necessary and sufficient for cavin-1 interaction. a Protein-equalised soluble cell extracts from HEK293 cells transfected with expression constructs encoding Flag-SOCS3 and myc-tagged cavin-1 as indicated were processed by immunoprecipitation (IP) with antiFlag M2-agarose beads prior to SDS-PAGE and immunoblotting with the indicated antibodies. Whole-cell lysates from the samples used in the IP were also fractionated by SDS-PAGE for immunoblotting in parallel. b Protein-equalised soluble cell extracts from WT and SOCS3-null AS-M.5 cells treated with 50 $\mu \mathrm{M}$ Fsk and $6 \mu \mathrm{M}$ MG132 for $4 \mathrm{~h}$ were processed by IP with anti-cavin-1 antibody prior to SDS-PAGE and immunoblotting with the indicated antibodies. As loss of SOCS3 in AS-M.5 cells reduces cavin-1 protein levels (a), twice the amount of protein was used as input for SOCS3-null cells to compensate. NS = non-specific band. c Upper: Protein-equalised soluble cell extracts from HEK293 cells transfected with expression constructs encoding either Flag-tagged WT SOCS3 or the indicated truncation mutants and GFP-tagged cavin-1 as indicated were processed by IP with anti-Flag M2-agarose beads prior to SDSPAGE and immunoblotting with the indicated antibodies. Whole-cell lysates from the same samples used in the IP were also fractionated by SDS-PAGE for immunoblotting in parallel. Lower: Schematic of the SOCS3 truncation constructs used, KIR = kinase inhibitory region, ESS $=$ extended SH2 subdomain, PEST = PEST (ProGluSerThr) motif. $\mathbf{d}$ Upper: Protein-equalised soluble cell extracts from HEK293 cells co-transfected with expression constructs encoding either full-length SOCS3 (WT), a truncated $\triangle \mathrm{SH} 2$ SOCS3domain $(\triangle \mathrm{SH} 2)$, or SOCS box domain (SB) fused to GFP and myc-tagged cavin-1 as indicated were processed by IP with anti-GFP antibody and protein G-Sepharose beads prior to SDS-PAGE and immunoblotting with the indicated antibodies. Wholecell lysates from the same samples used in the IP were also fractionated by SDS-PAGE and for immunoblotting in parallel. Lower: Schematic of the SOCS3GFP fusions used

interaction with cavin-1, we next examined whether the PEST sequence insert was involved. To do this, we utilised a $\triangle \mathrm{PEST}$ SOCS3 deletion mutant in which the PEST motif (Pro129Arg163) was removed and replaced with (Gly-Ser) $x 4^{36}$. $\triangle$ PEST SOCS3 was expressed at comparable levels to WT SOCS3 in transfected HEK293 cells and, consistent with previously published work $^{33}$, replacement of the PEST sequence did not diminish specific interaction with PTyr759 peptide as determined by in vitro peptide pull-down assays (Fig. 5a). In contrast, the ability of $\triangle$ PEST SOCS3 to bind cavin-1 in co-IP experiments was almost completely lost (Fig. 5b), thus demonstrating that the SOCS3 SH2 domain PEST insert was specifically required for cavin-1 interaction. Additional co-IP experiments using CIS, which also has a PEST insert in its $\mathrm{SH} 2$ domain, revealed that it was also able to form a complex with cavin-1 in co-transfected cells (Fig. 5c). Next we sought to determine whether the SOCS3 


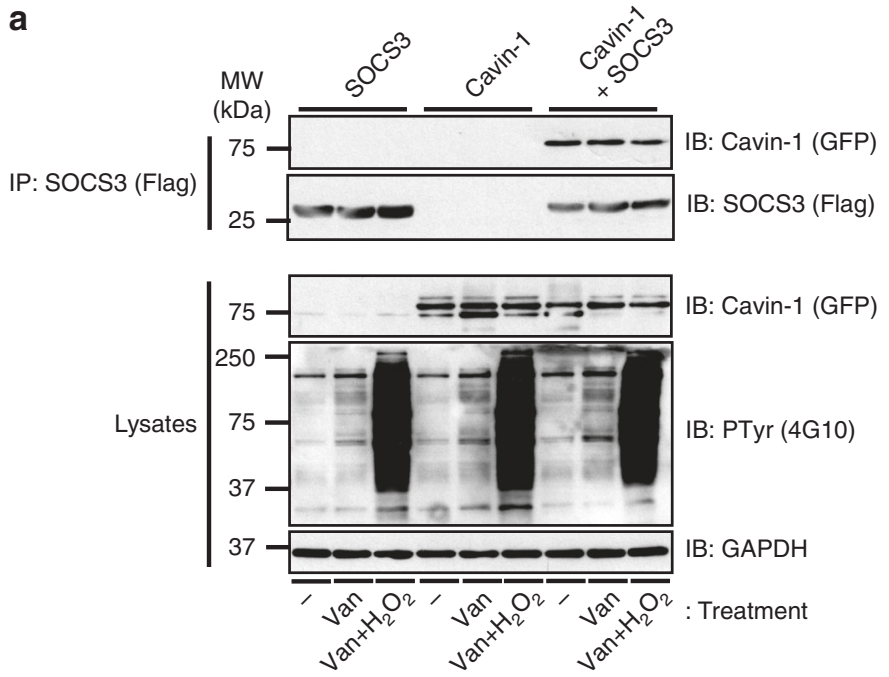

b

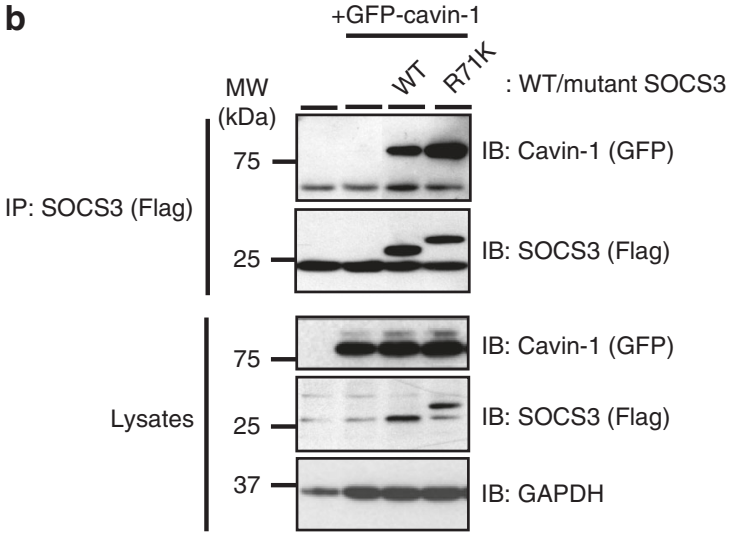

C
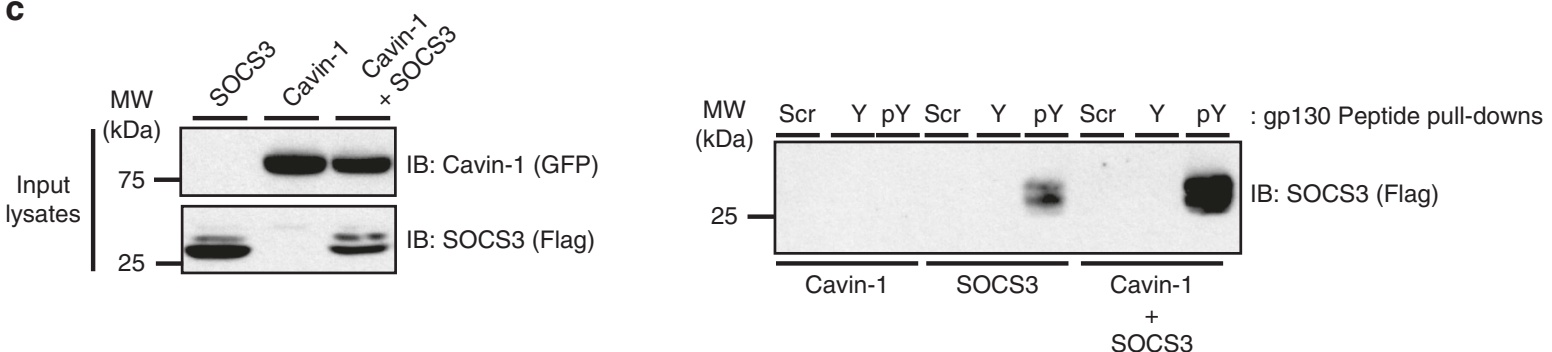

Fig. 4 Cavin-1-SOCS3 interaction occurs independently of the PTyr binding capacity of the SH2 domain. a HEK293 cells transfected with expression constructs encoding Flag-SOCS3 and GFP-tagged cavin-1 as indicated were treated with or without Tyr phosphatase inhibitors sodium orthovanadate (Van: $1 \mathrm{mM})$ for $1.5 \mathrm{~h}$ and then hydrogen peroxide $\left(\mathrm{H}_{2} \mathrm{O}_{2}: 0.2 \mathrm{mM}\right)$ for an additional $30 \mathrm{~min}$ prior to harvesting. Protein-equalised soluble cell extracts were then processed by IP with anti-Flag M2-agarose beads prior to SDS-PAGE and immunoblotting with the indicated antibodies. Whole-cell lysates from the samples used in the IP were also fractionated by SDS-PAGE for immunoblotting in parallel. b Protein-equalised soluble cell extracts from HEK293 cells transfected with expression constructs encoding either WT or R71K-mutated Flag-SOCS3 and GFP-tagged-cavin-1 as indicated were processed by IP with anti-Flag M2-agarose beads prior to SDS-PAGE and immunoblotting with the indicated antibodies. Whole-cell lysates from the samples used in the IP were also fractionated by SDS-PAGE for immunoblotting in parallel. c Protein-equalised soluble cell extracts from HEK293 cells transfected with expression constructs encoding WT Flag-SOCS3 and GFP-tagged cavin-1 as indicated were incubated with $100 \mathrm{nM} \mathrm{N}$-terminally biotinylated peptides corresponding to the Tyr759 motif of gp130 in its phosphorylated (pY) or non-phosphorylated (Y) forms or a scrambled control (Scr) and streptavidin-agarose beads prior to SDS-PAGE and immunoblotting with the indicated antibodies. Whole-cell lysates from the samples used in the pull-down were also fractionated by SDS-PAGE for immunoblotting in parallel

PEST sequence was sufficient to confer interaction with cavin-1. Bioinformatic analysis using ePESTfind (http://emboss. bioinformatics.nl/cgi-bin/emboss/epestfind) identified human Grap2 as a candidate $\mathrm{SH} 2$ domain-containing protein that lacked a detectable PEST sequence. In addition, Grap2 was unable to form a complex with cavin-1 upon co-expression in transfected HEK293 cells (Fig. 5d). Therefore, Pro129-Arg163 from SOCS3 was transplanted onto the central Grap2 $\mathrm{SH} 2$ domain and the resulting chimera (Grap2-S3PEST) assessed for its ability to form a complex with co-expressed cavin-1 in transfected HEK293 cells. These experiments demonstrated that insertion of SOCS3 PEST sequence was sufficient to confer an ability to bind cavin-1 on the resulting Grap2-S3PEST chimera, albeit to a much weaker extent than WT SOCS3 (Fig. 5d).

Multiple cavin-1 regions required for SOCS3 interaction. To examine whether SOCS3 interacts directly with cavin-1, peptide arrays of overlapping 25-mer peptides sequentially shifted by five amino acids and spanning the full-length cavin-1 open reading frame were overlaid with purified recombinant SOCS3 and visualised by probing with anti-SOCS3 antibodies (Fig. 6a). Dark spots represent positive areas of SOCS3 interaction. Using this approach we found that SOCS3 could interact strongly with two distinct regions spanning $>70$ amino acids within the cavin-1 open reading frame: an $\mathrm{N}$-terminal region spanning residues 75-152 and a C-terminal region encompassing residues 200-295. To validate the importance of these regions in controlling interaction with SOCS3 in intact cells, we generated a panel of myc epitope-tagged $\mathrm{N}$ - and C-terminal truncation mutants of cavin-1 (Fig. 6b) and tested their ability to co-IP Flag-SOCS3 upon coexpression in transfected HEK293 cells. All the truncated cavin-1 mutants expressed comparably to WT cavin-1 except for the C1 construct encoding residues 1-75 (Fig. 6c). These experiments demonstrated that, compared with WT cavin-1, each of the Nterminal and C-terminal truncation mutants was compromised in its capacity to co-IP with SOCS3. In conjunction with data from the peptide array experiments, our findings demonstrate that multiple SOCS3 binding interfaces within cavin-1 were required for optimal interaction with SOCS3.

Cavin-1 promotes SOCS3 localisation to the plasma membrane. Cavin-1 is required for stabilisation and maturation of caveolae at the plasma membrane, although it is also present with caveolin-1 in non-lipid raft fractions ${ }^{37}$. SOCS3 is thought to be 
a

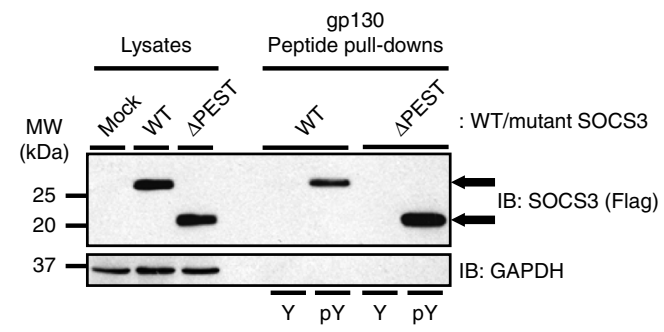

C

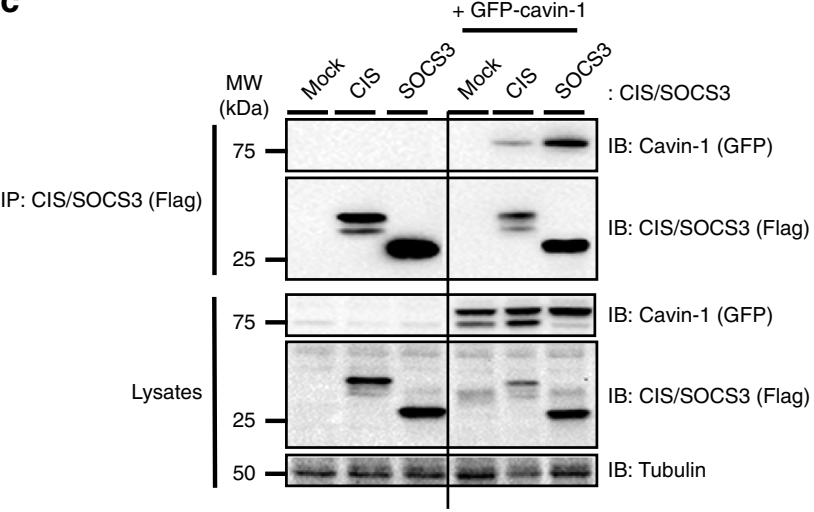

b
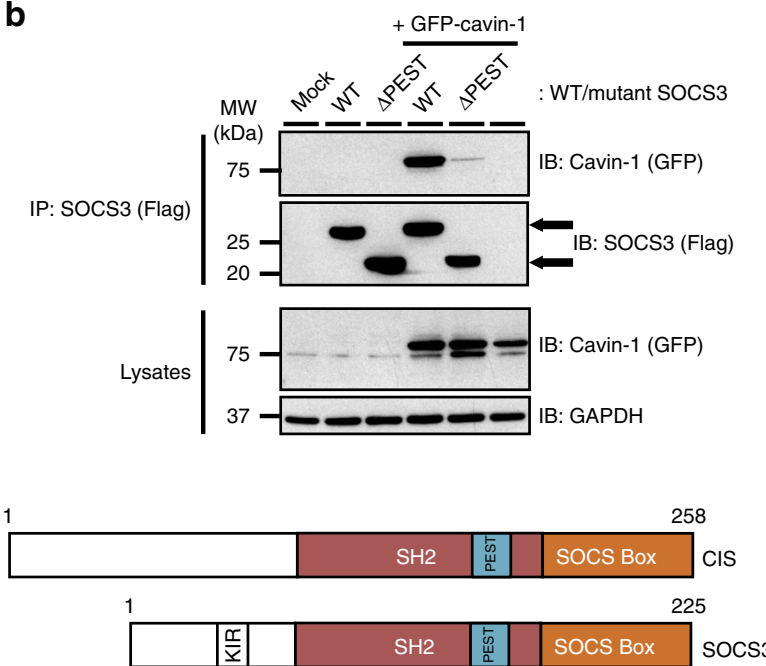

d + GFP-cavin-1

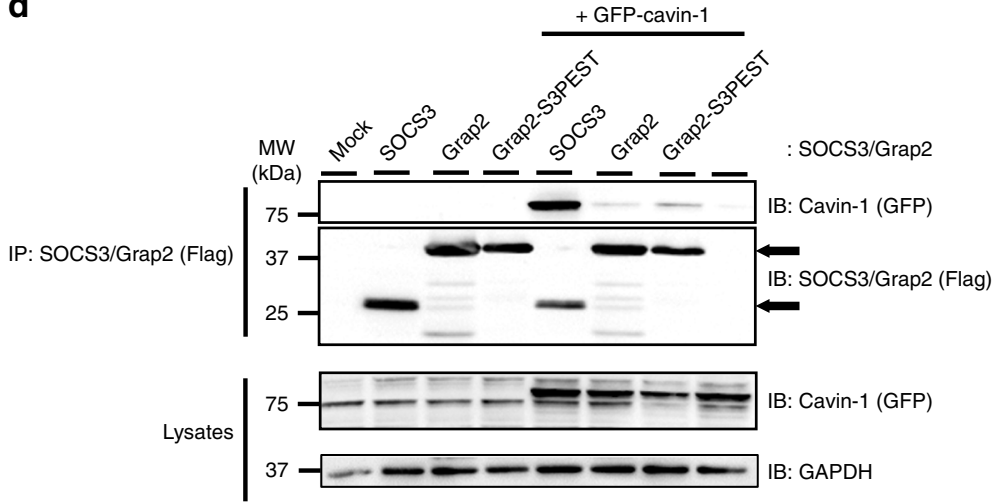

Fig. 5 Cavin-1-SOCS3 interaction requires the SOCS3 SH2 PEST motif. a Protein-equalised soluble cell extracts from HEK293 cells transfected with expression constructs encoding either WT or $\triangle$ PEST Flag-SOCS3 were incubated with $100 \mathrm{nM} \mathrm{N}$-terminally biotinylated peptides corresponding to the Tyr759 motif of gp130 in its phosphorylated ( $p Y$ ) or non-phosphorylated (Y) forms and streptavidin-agarose beads prior to loading with whole-cell lysate samples for SDS-PAGE and immunoblotting with the indicated antibodies. b Protein-equalised soluble cell extracts from HEK293 cells transfected with expression constructs encoding either WT or $\triangle$ PEST Flag-SOCS3 and GFP-tagged cavin-1 as indicated were processed by IP with anti-Flag M2-agarose beads prior to SDS-PAGE and immunoblotting with the indicated antibodies. Whole-cell lysates from the samples used in the IP were also fractionated by SDS-PAGE for immunoblotting in parallel. c Upper, Protein-equalised soluble cell extracts from HEK293 cells transfected with or without the indicated CIS and SOCS3 expression constructs and GFP-tagged cavin-1 as indicated were processed by IP with anti-Flag M2-agarose beads prior to SDS-PAGE and immunoblotting with the indicated antibodies. Whole-cell lysates from the samples used in the IP were also fractionated by SDS-PAGE for immunoblotting in parallel. Lower, Schematic of CIS and SOCS3. d Protein-equalised soluble cell extracts from HEK293 cells transfected with or without the indicated SOCS3 and Grap2 expression constructs and GFP-tagged cavin-1 as indicated were processed by IP with anti-Flag M2-agarose beads prior to SDS-PAGE and immunoblotting with the indicated antibodies. Whole-cell lysates from the samples used in the IP were also fractionated by SDS-PAGE for immunoblotting in parallel

recruited to activated cytokine receptors at the plasma membrane following the formation of a SOCS3 interaction motif upon phosphorylation of key $\mathrm{Tyr}$ residues by cytokine-activated $\mathrm{JAKs}^{19}$. Therefore, to examine a role for cavin-1 in controlling SOCS3 localisation, we used confocal microscopy to assess the effect of cavin-1 deletion on the subcellular distribution of a SOCS3-GFP fusion protein expressed in transfected cells plated at low density. A transfected SOCS3-GFP construct was used for these experiments as we failed to specifically detect endogenous SOCS3 staining in WT MEFs over and above background staining in $\mathrm{SOCS}^{-/-}$MEFs in confocal imaging experiments using three separate commercially available antibodies. In transfected WT MEFs, two populations of SOCS3-GFP-derived fluorescence were detectable: a punctate intracellular pool and a plasma membrane-localised pool (Fig. 7a). Endogenous cavin-1 was localised predominantly at the plasma membrane of the trailing edge of the cells as described by others ${ }^{38}$. Merging of the images revealed co-localisation of SOCS3-GFP and cavin-1 specifically at the plasma membrane (Fig. 7a). Analysis of SOCS3GFP/cavin-1 staining produced Pearson's correlation coefficient 
a

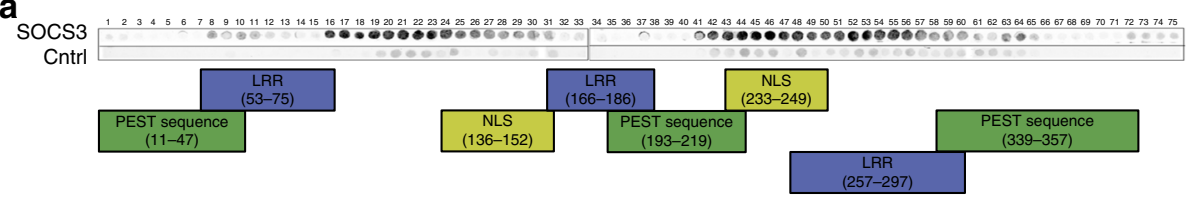

b
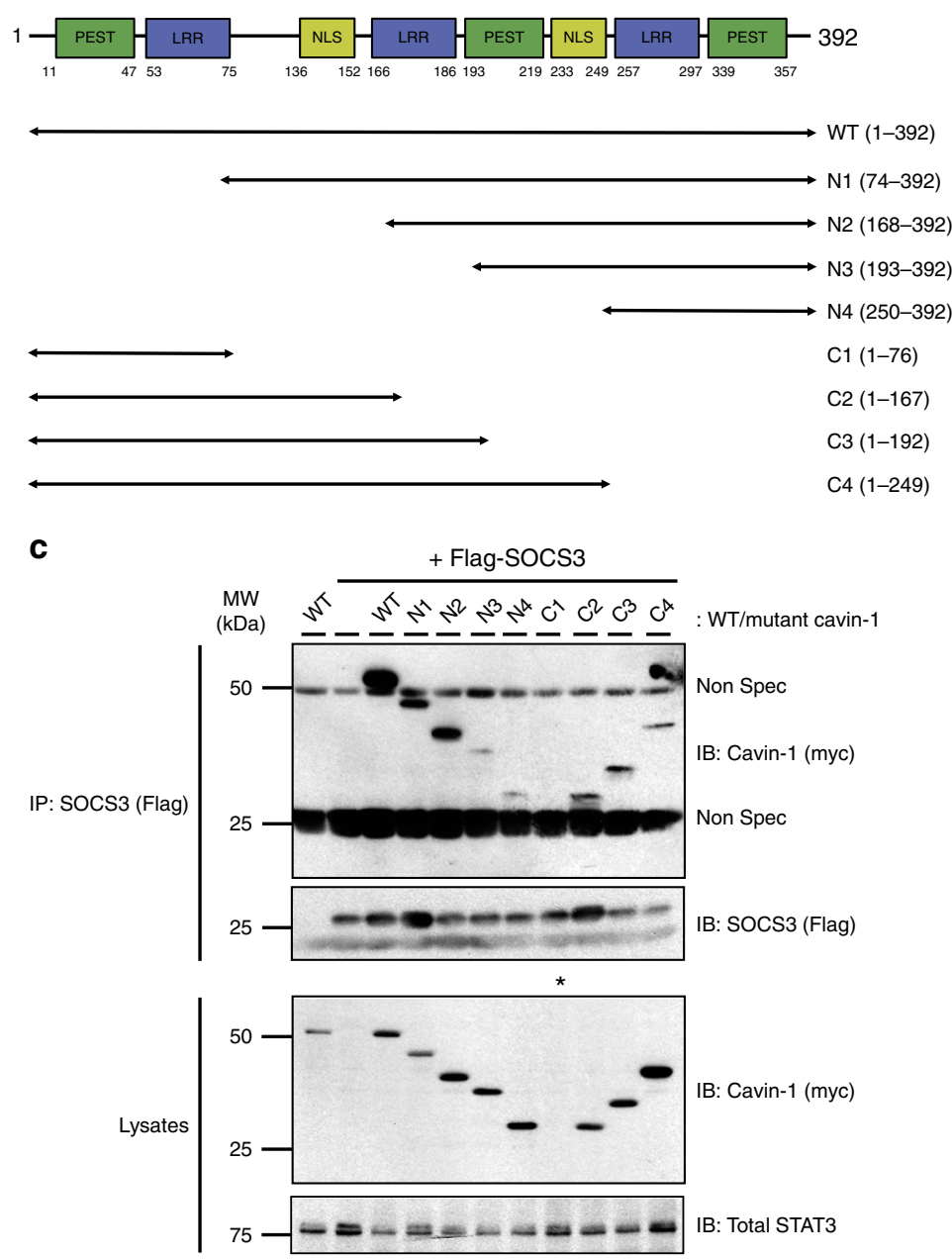

Fig. 6 SOCS3 interacts with multiple regions within cavin-1. a An immobilised library of 25-mer peptides sequentially shifted by 5 amino acids along the entire cavin-1 open reading frame was overlaid with either purified SOCS3 or a negative control (Cntrl). Dark spots represent areas of interaction between SOCS3 and peptides within the cavin-1 peptide array. The domain structure of murine cavin-1 is indicated below the overlay. $\mathbf{b}$ Schematic representation of the $\mathrm{N}$ - and C-terminally truncated myc-tagged cavin-1 mutants used for co-IP experiments. c Protein-equalised soluble cell extracts from HEK293 cells transfected with expression constructs encoding either myc-tagged WT cavin-1 or the indicated truncation mutants and Flag-tagged SOCS3 as indicated were processed by IP with anti-Flag M2-agarose beads prior to SDS-PAGE and immunoblotting with the indicated antibodies. Whole-cell lysates from the same samples used in the IP were also fractionated by SDS-PAGE for immunoblotting in parallel. *Indicates that expression of the C1 cavin-1 mutant was not detectable; Non Spec refers to immunoglobulin-derived non-specific staining

values of $>0.90$ at the plasma membrane, indicative of a high degree of co-localisation (Fig. 7b). Conversely, in cavin-1 $1^{-1-}$ MEFs SOCS3-GFP was undetectable at the plasma membrane and only present within a punctate intracellular pool (Fig. 7a). Importantly, transient co-expression of SOCS3-GFP with cotransfected cavin-1-mCherry into cavin-1 ${ }^{-1-}$ MEFs was able to restore their co-localisation at the plasma membrane (Fig. 7c). Expression of GFP alone in WT MEFs did not produce any detectable co-localisation with cavin-1, and its distribution was similar in both WT and cavin-1 $1^{-1-}$ MEFs (Supplementary Fig. 5A, B).

Additionally, subcellular fractionation experiments demonstrated that cavin-1 was mainly present in membrane and cytoplasmic fractions. This mirrored the subcellular distribution of SOCS3 in WT MEFs following induction by Fsk treatment for $5 \mathrm{~h}$. Interestingly, cavin-1 deletion shifted the distribution of SOCS3 predominantly to the cytoplasm (Fig. 7d). Thus, the presence of cavin-1 was important for localising endogenous SOCS3 to the membrane fraction, consistent with our confocal imaging experiments using SOCS3-GFP (Fig. 7a-c). Subcellular fractionation experiments also demonstrated that SOCS3 deletion produced a comparable reduction in caveolin-1 expression at the membrane as deletion of cavin-1, indicative of an indirect role for SOCS3 in maintaining caveolin-1 expression via stabilisation of cavin-1 (Fig. 7d). This change was specific for caveolin-1 as levels of the membrane marker flotillin were unaffected by deletion of either SOCS3 or cavin-1 (Fig. 7d). Therefore, together these data indicate that cavin-1 co-localised with a plasma membrane pool 
a $_{\text {Cavin }^{-1++}}$

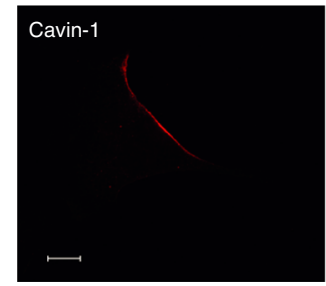

Cavin-1 ${ }^{-1-}$

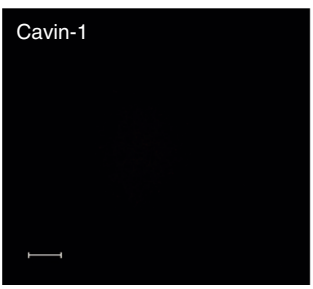

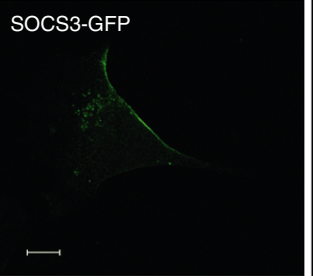

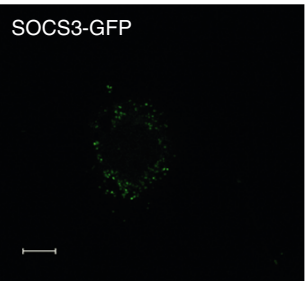

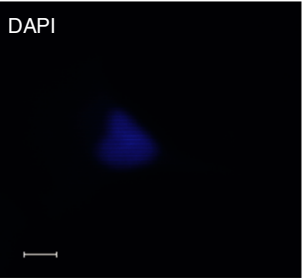
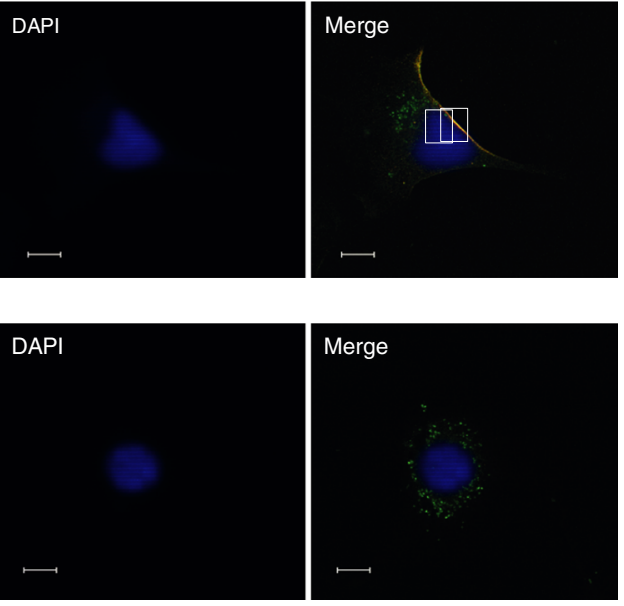

b
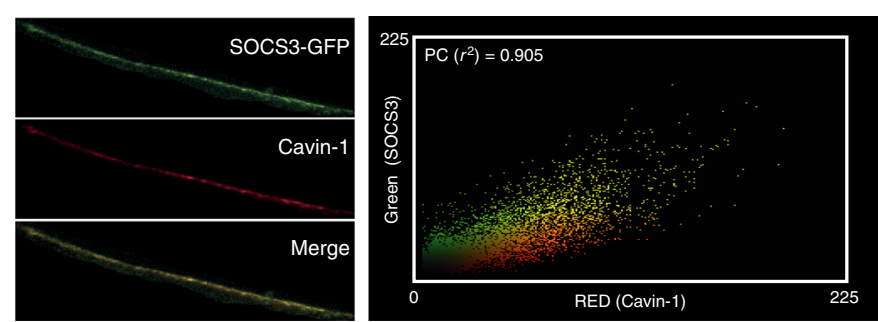

C
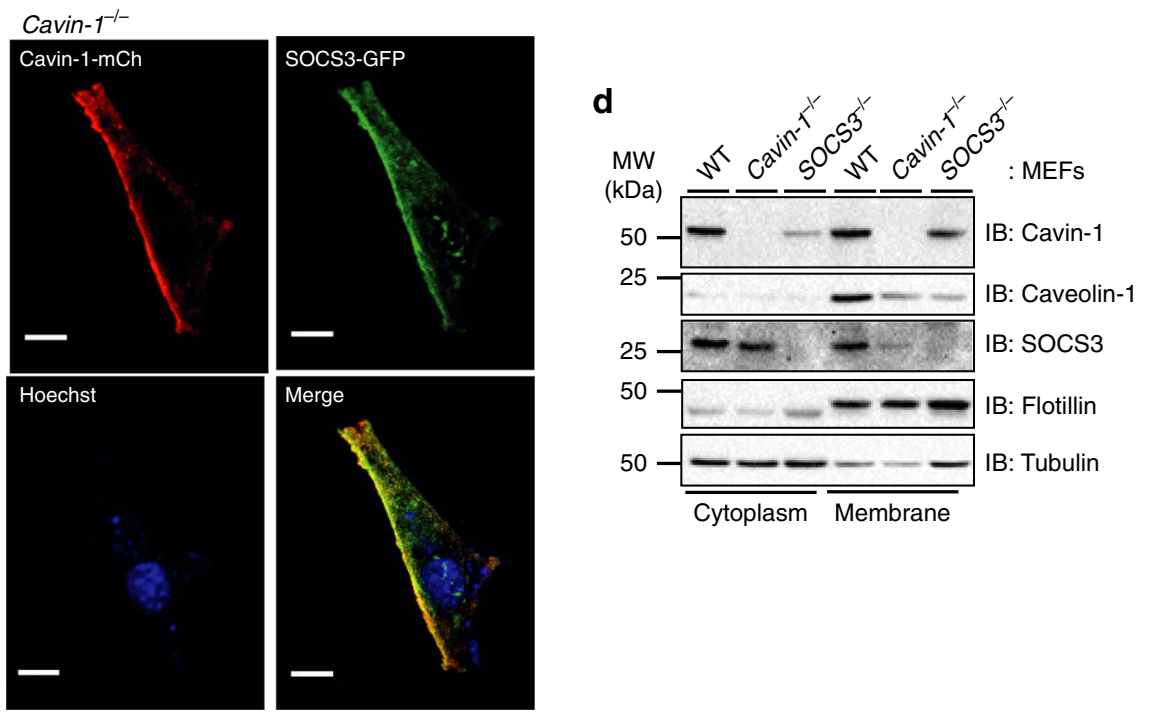

Fig. 7 Cavin-1 drives SOCS3 localisation to the plasma membrane. a WT (cavin-1 ${ }^{+/+}$) and cavin-1-/- MEFs transiently expressing SOCS3-GFP (green) were stained with DAPI prior to being fixed, solubilised, and stained with anti-cavin-1 antibody (red) before mounting for imaging by confocal microscopy. Areas of red and green overlap are yellow. Scale bar $=10 \mu \mathrm{m}$. b Pearson's correlation coefficient value $\left(r^{2}\right)$ measured from the intensity values located within the rectangular region on the plasma membrane superimposed on the merged cavin-1/SOCS3-GFP image from WT MEFs. c Cavin-1-/- MEFs transiently coexpressing SOCS3-GFP (green) and cavin-1-mCh (red) were stained with Hoechst 33342 prior to being fixed for imaging by confocal microscopy. Areas of red and green overlap are yellow. Scale bar $=10 \mu \mathrm{m}$. d WT, SOCS3 ${ }^{-/}$, and cavin-1-/- MEFs were pre-incubated with Fsk (50 $\mu \mathrm{M}$ ) for $5 \mathrm{~h}$ to induce SOCS3 prior to subcellular fractionation and analysis by SDS-PAGE and immunoblotting with the indicated antibodies

of SOCS3 in intact cells and was an important determinant of SOCS3 localisation to the plasma membrane.

Cavin-1 limits IL-6-stimulated Tyr705 STAT3 phosphorylation. While some studies have demonstrated localisation of cytokine receptors and JAKs in lipid raft microdomains ${ }^{15-18}$, relatively little is known about the impact of caveolin expression/ function on JAK-STAT signalling and no studies have specifically examined a role for cavins. Our data suggested that cavin-1 and SOCS3 interacted directly and co-localised at the plasma membrane, while SOCS3 was mainly cytosolic in the absence of cavin1. To examine any functional impact of cavin-1 on cytokine signalling, we examined the effects of cavin-1 deletion in MEFs on IL-6-mediated activation of STAT3, as determined by phosphorylation at Tyr705 which is required for STAT3 to form 
transcriptionally active complexes ${ }^{39}$. While stimulation of both WT and cavin- $1^{-1-}$ MEFs with a sIL-6R $\alpha /$ IL- 6 trans-signalling complex triggered a transient increase in STAT3 phosphorylation on Tyr705, the response was greater and more sustained in cavin$1^{-/-}$MEFs, being detectable at the 60 and 120 min time points in cavin $1^{-1-}$ but less pronounced in WT cells (Fig. 8a). Interestingly, Tyr705 phosphorylation was specifically enhanced as STAT3 phosphorylation on Ser727 (which is mediated by several candidate Ser/Thr kinases ${ }^{40}$ ) was unaffected by cavin-1 deletion. Moreover, the increase in IL-6 signalling occurred despite reduced levels of JAK1 in cavin-1 ${ }^{-/-}$MEFs, although this reduction did not reach statistical significance (Fig. 8a). Other cytokine receptor complexes that utilise gp130 include those for leukaemia inhibitory factor (LIF) and oncostatin M (OSM): LIF signals via gp130/LIF receptor (LIFR) heterodimers, while OSM signals downstream using either LIFR/gp130 or OSM receptor/ gp130 complexes $^{41}$. As observed with sIL-6R $\alpha /$ IL-6, Tyr705 phosphorylation of STAT3 in response to either LIF or OSM was greater in cavin $-1^{-/-}$versus WT MEFs at 60 min (Fig. 8b). Taken together, these data suggested that loss of cavin-1 compromised one or more inhibitory mechanisms responsible for suppressing gp130- and JAK-mediated Tyr phosphorylation of STAT3.

Previous studies have demonstrated that depletion or loss of SOCS3 results in prolonged activation of STAT3 in response to specific cytokines ${ }^{42-44}$, similar to the effect observed upon cavin-1 deletion. We have shown previously that the ability of cAMP to inhibit IL-6 signalling in vascular ECs, MEFs, and COS cells has an absolute requirement for Epac1-dependent induction of $\mathrm{SOCS}^{26,27,45}$. Given the importance of cavin-1 in localising SOCS3 to the plasma membrane and the sustained phosphorylation of STAT3 on Tyr705 observed following sIL-6R $\alpha /$ IL6 stimulation of cavin-1 $1^{-1}$ MEFs, we examined the impact of cavin-1 deletion on the inhibitory effect of cAMP which has previously been shown to be SOCS3 dependent ${ }^{26,45}$. These experiments demonstrated that while pre-treatment of WT MEFs with cAMP-elevating drug Fsk $(50 \mu \mathrm{M})$ significantly inhibited sIL-6R $\alpha /$ IL-6-stimulated Tyr705 phosphorylation of STAT3, this effect was lost in cavin-1 ${ }^{-1-}$ MEFs even though Fsk in combination with sIL-6R $\alpha /$ IL-6 produced equivalent levels of SOCS3 in WT and cavin-1 ${ }^{-1-}$ MEFs (Fig. 8c). These results also did not reflect a non-specific reduction in cAMP responsiveness following loss of cavin-1 as Fsk could trigger the accumulation of cAMP target gene Nur77 equivalently in both WT and cavin-1 ${ }^{-/-}$ MEFs (Supplementary Fig. 6). Therefore, the presence of cavin-1 was necessary for SOCS3-mediated inhibition of IL-6 signalling by cAMP.

\section{Discussion}

The importance of SOCS3 in limiting downstream signalling from cytokine receptor complexes that utilise gp130, as well as the leptin receptor $\mathrm{ObRb}$ and the G-CSFR, is well established ${ }^{6,7}$ However, relatively little is known about how SOCS3 interaction with other intracellular proteins can impact on its ability to inhibit signalling. As part of a study to identify SOCS3-regulated substrates, we performed "stable isotopic labelling of amino acids in cell culture" (SILAC) analysis of ubiquitinome profiles in WT and $\mathrm{SOCS}^{-1}$ MEFs stably expressing a tandem affinity purification (TAP)-tagged ubiquitin transgene ${ }^{46}$. Using this approach, the caveola scaffolding protein cavin-1 was identified as a ubiquitinated protein whose levels were stabilised in WT cells. We have demonstrated that cavin-1 can interact with SOCS3 and that the absence of SOCS3 results in increased turnover of cavin-1 and a parallel reduction in cellular levels of caveolin-1 and cell surface caveloae. We have also demonstrated that cavin-1 is important for effective SOCS3-mediated suppression of
JAK-STAT signalling in response to sIL-6R $\alpha / \mathrm{IL}-6$ trans-signalling complexes.

The importance of caveolae and other lipid raft microdomains for maintaining signalling from the plasma membrane has been demonstrated for a variety of systems, including endothelial nitric oxide synthase and $\mathrm{Src}^{47,48}$. In comparison, relatively little information is available on how they regulate JAK-STAT pathway activation. Localisation of JAK-STAT signalling components, including gp130, receptors for growth hormone, ciliary neurotrophic factor and LIF, and JAK2 to lipid rafts has been determined by biochemical fractionation of cell extracts ${ }^{15-18,49-52}$. However, the functional consequences appear to be context dependent, such that raft disruption by treatment with cholesterol-depleting agents like $\beta$-cyclodextrin or homozygous deletion of caveolin-1 can either inhibit ${ }^{15,16,49}$ or enhance ${ }^{52,53}$ downstream signalling. Thus, Lisanti and colleagues $^{52}$ have examined the effects of manipulating caveolin-1, and demonstrated that caveolin-1 can suppress prolactin receptor-mediated JAK2-dependent phosphorylation and activation of STAT5a in murine mammary epithelial cells in vitro, consistent with observations that caveolin-1 deletion in vivo enhances prolactin receptor signalling ${ }^{53}$. The mechanism proposed was via a direct interaction between caveolin-1 and JAK2, although no evidence of a direct effect of caveolin-1 on JAK2 Tyr kinase activity was presented $^{52}$. Other studies have specifically examined the importance of caveolae for gp130 function, demonstrating that a significant proportion of cellular gp130 resides in detergentresistant lipid rafts and can co-IP with caveolin-1. In addition, cholesterol depletion with $\beta$-cyclodextrin has been shown to trigger the re-distribution of gp130 to non-raft factions and attenuate the ability of IL-6 to stimulate STAT3 phosphorylation on $\operatorname{Tyr} 705^{16}$. In contrast, others have found that both gp130 and STAT3 are localised to lipid rafts ${ }^{15}$ and demonstrated an inverse relationship between caveolin-1 expression and STAT3 activation ${ }^{54}$. Therefore, while a weak association between membrane microdomains and JAK-STAT signalling modules has been made, the molecular mechanisms responsible for this interaction remain unclear.

Our data would suggest a novel route through which caveola accessory protein cavin-1 can modulate cytokine receptor signalling via interaction with the inhibitory regulator SOCS3. While SOCS3 expression is induced in response to many stimuli, conditional gene targeting strategies have revealed that sensitivity to SOCS3 is restricted to a panel of plasma membrane-localised cytokine receptors ${ }^{6,7,41}$. Consistent with another study ${ }^{38}$, we found that while cavin-1 was localised to the plasma membrane in WT MEFs, it was not distributed uniformly, instead localising to the trailing edge of migrating cells. Importantly, a significant proportion of SOCS3-GFP co-localised to the same plasma membrane compartment in WT but not cavin-1 ${ }^{-/-}$MEFs. Together with data showing that cavin-1 could co-IP with SOCS3 and that purified SOCS3 could interact with multiple cavin-1derived peptides in vitro, we propose that cavin-1 binds SOCS3 directly and that this contributes to efficient SOCS3 recruitment to the plasma membrane where it can effectively bind and inhibit cytokine receptors such as gp130. A key aspect of this model (Fig. 9) is that SOCS3 can still bind Tyr phosphorylated peptide in vitro in the presence of cavin-1. Interestingly, the SOCS3 SH2 domain appeared to fulfil both PTyr and cavin-1 binding functions as cavin-1 interaction required the PEST motif present within the SOCS3 SH2 domain, which we and others have shown to be dispensable for PTyr binding ${ }^{33,36}$. In some respects, this is similar to the recently described interaction between SOCS3 and CUEDC2, which also binds the SH2 domain and enhances SOCS3-mediated inhibition of JAK1-STAT3 activation by IL- $6^{11}$. Since CUEDC2 potentiates SOCS3 function it would be 
a
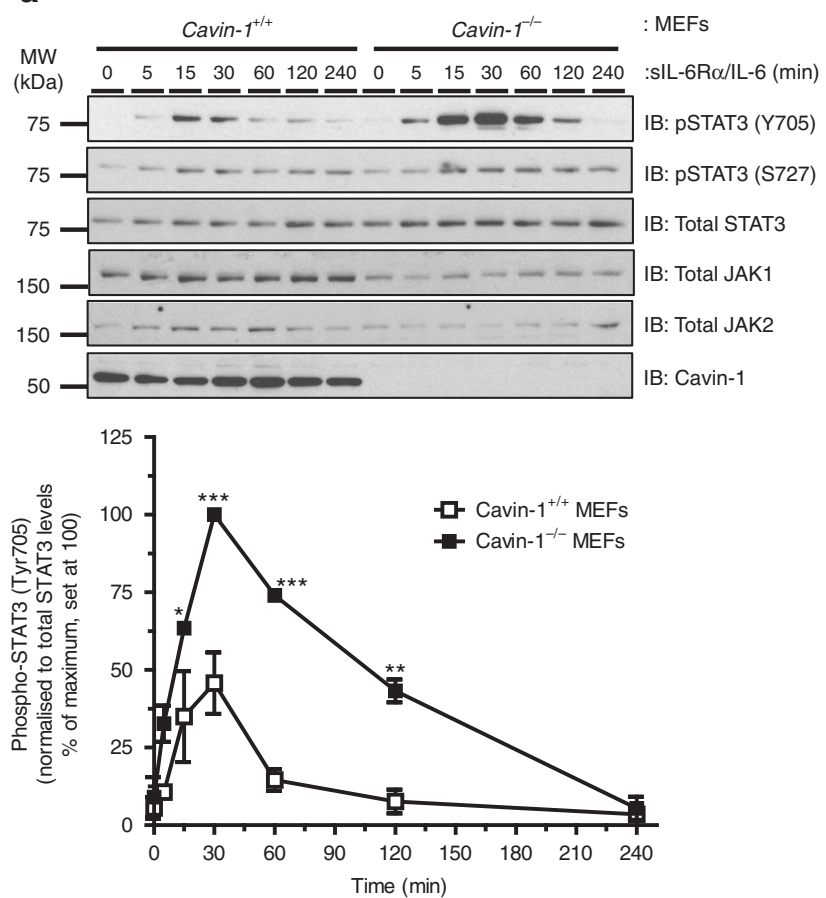

b

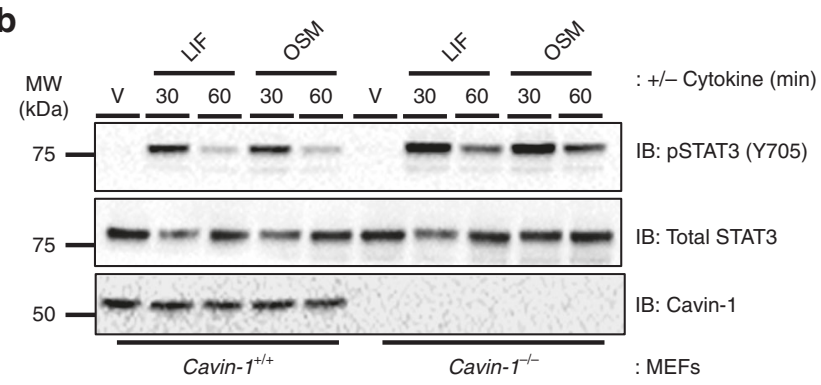

C
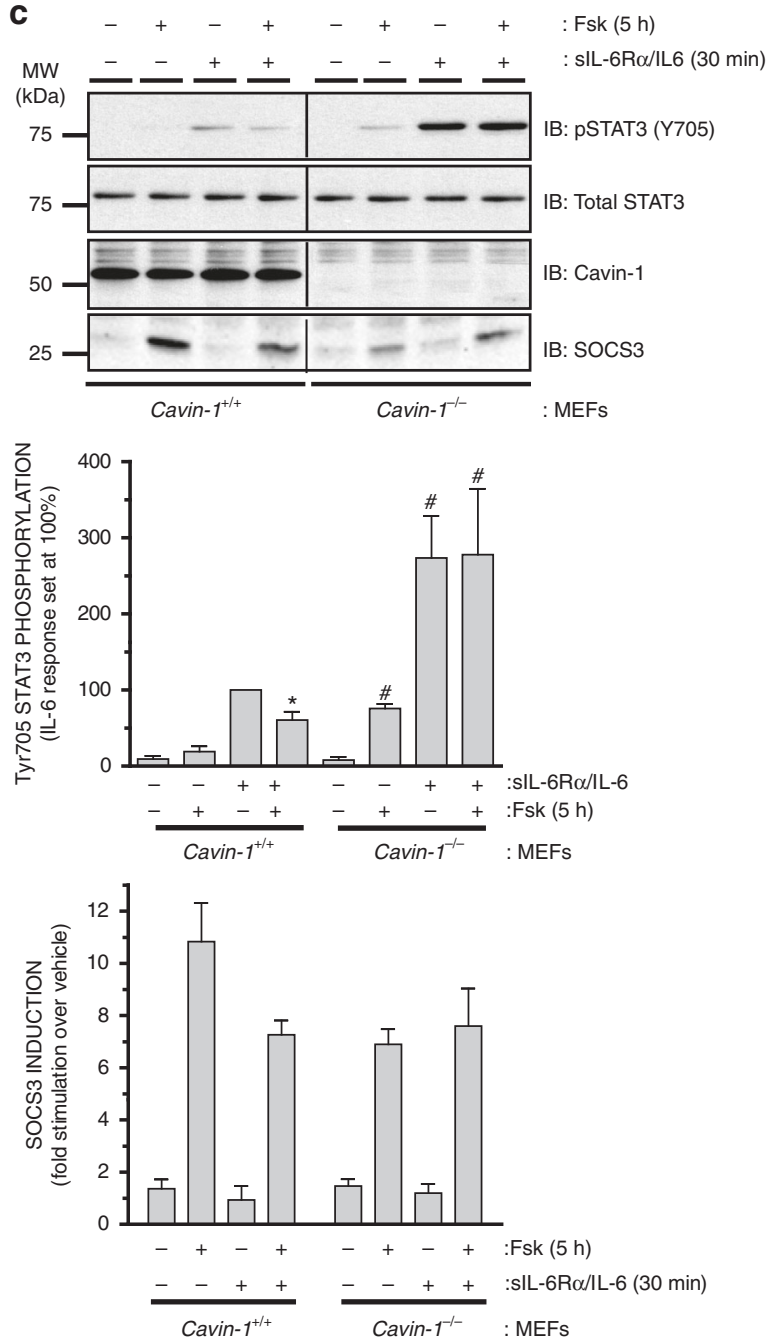

Fig. 8 Cavin-1 limits Tyr705 phosphorylation of STAT3 via SOCS3. a Upper: Protein-equalised soluble cell extracts from cavin-1 $1^{+/+}$and cavin-1-/- MEFs treated for the indicated times with sIL-6R $/ \mathrm{IL}-6\left(25\right.$ and $\left.5 \mathrm{ng} \mathrm{ml}^{-1}\right)$ were fractionated by SDS-PAGE prior to immunoblotting with the indicated antibodies. Quantitation of normalised Tyr705 phospho-STAT3 in cavin-1+/+ and cavin-1-/- MEFs are presented as mean values \pm standard error for $N=3$ experiments. ${ }^{\star} P<0.05,{ }^{\star \star} P<0.01,{ }^{\star \star \star} P<0.001$ vs. corresponding treatment in cavin-1 ${ }^{+/+}$MEFs. b Protein-equalised soluble cell extracts from cavin- ${ }^{+/+}$

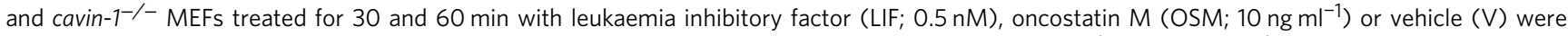
fractionated by SDS-PAGE prior to immunoblotting with the indicated antibodies. c Upper: WT $\left(\right.$ cavin $\left.-1^{+/+}\right)$and cavin-1 ${ }^{-/-}$MEFs were pre-incubated with or without Fsk $(50 \mu \mathrm{M})$ for $5 \mathrm{~h}$ prior to treatment with or without sIL-R $\alpha / \mathrm{IL}-6\left(25\right.$ and $\left.5 \mathrm{ng} \mathrm{ml}^{-1}\right)$ for 30 min. Cell extracts were analysed by SDS-PAGE and immunoblotting with the indicated antibodies. Lower: Quantitation of normalised Tyr705 phospho-STAT3 and SOCS3 in cavin-1 ${ }^{+/+}$and cavin-1 ${ }^{-/-}$MEFs is presented as mean \pm standard error for $N=3$ experiments, ${ }^{\star} P<0.05$ vs. sIL- 6 R $\alpha /$ IL- 6 treatment in cavin $-1^{+/+}$MEFs, ${ }^{\#} P<0.05$ vs. vehicle treatment in cavin-1 ${ }^{-/-}$MEFs.

anticipated that, like cavin-1, its interaction with the SH2 domain must be independent of PTyr binding, suggesting it may also involve the PEST sequence. However, in contrast to cavin-1, CUEDC2 localises to the cytoplasm and nucleus ${ }^{55}$. Nevertheless, our observations and those of Zhang et al. ${ }^{11}$ raise the possibility that multiple proteins may bind within the SOCS3 SH2 domain to facilitate localisation with Tyr phosphorylated binding partners in distinct subcellular compartments. In this regard, it should be noted that confocal imaging and subcellular fraction experiments detected SOCS3 in the cytoplasm as well as the plasma membrane, and that cavin-1 deletion resulted in the specific loss of the plasma membrane pool.

To date, we are only aware of one other study which has examined the impact of the PEST sequence on SOCS3 function ${ }^{36}$. However, these experiments were performed in HEK293 cells cotransfected to express a STAT3-responsive reporter gene and increasing amounts of either WT or $\triangle$ PEST SOCS3. The authors noted that at maximal levels of WT and $\triangle$ PEST SOCS3 expression, both constructs abolished LIF-stimulated activation of STAT3. However, upon normalising SOCS3 function with the expression levels of WT and $\triangle$ PEST SOCS3, they also noted that at submaximal expression levels the functionality of $\triangle \mathrm{PEST}$ SOCS3 was less than that of WT SOCS3. Thus, they concluded that WT SOCS3 is slightly more efficient at inhibiting STAT3 activation $^{36}$. Others have shown that low expression levels of SOCS3 inhibit signalling via interaction with g130 followed by inhibition of JAK activity, whereas overexpression SOCS3 can inhibit gp130 signalling independently of interaction with the SOCS3 binding site and works instead via direct inhibition of JAK $1^{19,56}$ These data would also suggest that any functional deficits in $\triangle$ PEST SOCS3 in localising to gp130 would be overcome by its overexpression. In contrast, our functional experiments examining signalling from endogenous proteins suggest an important aspect of SOCS3 PEST motif function is an interaction 


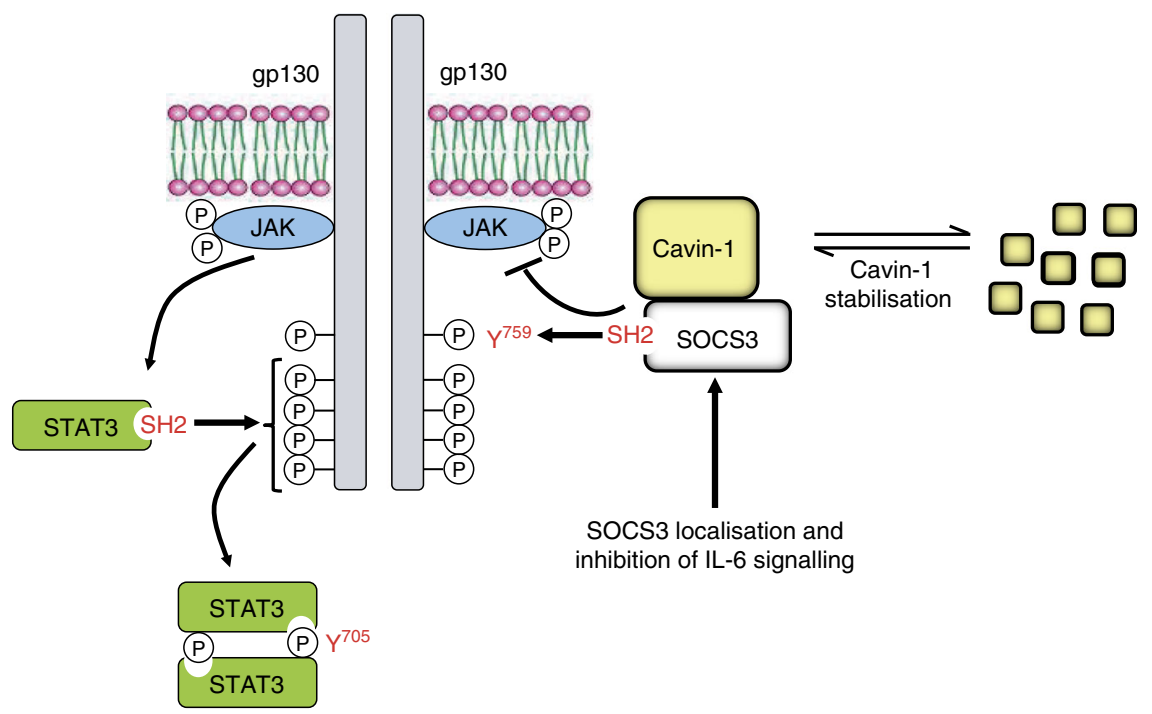

Fig. 9 Model of novel functional interactions between SOCS3 and cavin-1

with cavin-1 that is critical for effective regulation of JAK-STAT signalling. The effects on signalling of reconstituting cavin-1 $^{-/}$ MEFs with mutated cavin- 1 that fails to interact with SOCS3 but retains the ability to stabilise caveolin-1 would be very informative in dissecting whether cavin-1 is essential for SOCS3 function or simply enhances it through facilitating recruitment to the plasma membrane. It would also be important to assess any functional consequences of SOCS3 on gp130 ubiquitylation ${ }^{57}$ and receptor trafficking ${ }^{10}$ in order to fully assess the impact of cavin-1 on SOCS3 function.

While this adds an extra layer of regulation for SOCS3, our study has also identified a previously unknown mechanism by which SOCS3 can regulate cavin-1 function by enhancing its stability and, as a consequence, maintaining expression levels of caveolin-1 and cell surface caveolae. Similar observations have recently been reported for Eps15 homology domain-containing protein 2 (EHD2) which, to our knowledge, is the only other example of a cavin-1-interacting protein that regulates caveola stability, although a direct effect on cavin-1 turnover has not been examined ${ }^{58}$. More generally, our findings also raise the possibility that cavins constitute a new class of SOCS3-interacting proteins. While the presence of cavin-1 and caveolin-1 is sufficient to generate caveolae in many cell type ${ }^{59}$, MEFs also express cavin-2 and cavin-3. Elegant biochemical and biophysical studies have demonstrated that cavins assemble into oligomeric complexes both in cells and in vitro ${ }^{60,61}$. While each of the cavins is detectable on individual caveolae ${ }^{59}$, cavin-2 and cavin-3 appear to form distinct hetero-oligomeric complexes with cavin-1 rather than with each other ${ }^{60}$. Thus, it would be anticipated that SOCS3 should interact with both cavin-1/cavin-2 and cavin-1/ cavin-3 oligomers and therefore distribute itself uniformly around caveolar bulbs similarly to cavin $-1^{61}$. As the SOCS3 PEST sequence was necessary for cavin-1 interaction, it would also be informative to assess what extent this property is shared among similar sequences present in other SOCS family members. Analysis of the SOCS family revealed that CIS, which like SOCS3 can also interact with cavin-1, contains a PEST motif located in its SH2 domain, while SOCS1, SOCS5, and SOCS7 each have one or more PEST sequences located within their N-terminal domains. In contrast, no PEST motifs are found in SOCS2, SOCS4, and SOCS6. Given the distinct roles of different SOCS family members in regulating signalling ${ }^{62}$, the functional significance of the identified PEST motifs and their roles in determining the localisation of individual SOCS proteins via distinct protein interactions warrant further investigation.

Finally, our findings may have implications in the context of how cavin-1 and SOCS3 dysfunction can trigger disease. Several inactivating frameshift mutations within exon 2 of the cavin-1 gene that result in the production of altered cavin-1 proteins have been identified in patients with general lipodystrophy, muscular dystrophy, and insulin resistance ${ }^{63-66}$. In each case, a lack of functional cavin-1 is associated with the downregulation and/or mislocalisation of all three caveolin subtypes in skeletal muscle and an absence of cell surface caveolae in patient-derived fibroblast $^{64}$ and skeletal muscle ${ }^{63}$. As multiple regions within cavin-1 are required for optimal binding of SOCS3, the mutated cavin-1 proteins identified in patients with congenital generalised lipodystrophy would be predicted to be compromised in their ability to interact with SOCS3, thereby resulting in enhanced IL-6 responses. In this regard, cardiac-specific homozygous deletion of murine SOCS3 results in contractile dysfunction and the occurrence of a variety of ventricular arrhythmias ${ }^{67}$, the latter of which is also observed in patients with inactivating cavin- 1 mutations ${ }^{64}$. Finally, a F136L germline SOCS3 mutation found in a subset of polycythemia vera patients has been shown to display an impaired capacity to inhibit erythropoietin receptor-JAK2 signalling ${ }^{68}$. As F136 is located within the PEST insert we have identified as critical for cavin-1 interaction, this mutation may alter cavin-1 binding to SOCS3 to block its inhibitory effects on JAK-STAT signalling. Based on our findings, future studies will therefore need to examine how cavin-1 and/or SOCS3 mutations identified in patients interact to trigger defective regulation of signalling in these pathologies.

\section{Methods}

Cell culture and transfection. HEK293 cells were obtained from the European Collection of Authenticated Cell Cultures (ECACC) through Sigma. Immortalised SOCS3 $^{-/-}$and cavin-1 $1^{-1-}$ MEFs and the corresponding WT cell lines have been described previously ${ }^{59,69}$. HEK293 cells and MEFs were maintained in Dulbecco's modified Eagle's medium (DMEM) supplemented with $10 \%(\mathrm{v} / \mathrm{v})$ foetal bovine serum (FBS), $1 \mathrm{mM}$ L-glutamine, $100 \mathrm{U} \mathrm{ml}^{-1}$ penicillin and $100 \mu \mathrm{M}$ streptomycin. AS-M.5 human angiosarcoma-derived ECs generously provided by Dr Vera Krump-Konvalinkova and Professor Charles Kirkpatrick (Johannes Gutenberg University of Mainz, Germany) ${ }^{28}$ were cultured in endothelial growth medium-2 supplemented with $2 \%(\mathrm{w} / \mathrm{v})$ FBS, hydrocortisone, ascorbate, and recombinant growth factors as recommended by the supplier (Lonza). HEK293 cells at $80 \%$ confluence on poly-D-lysine-coated dishes were transfected with 2-8 $\mu$ g of complementary DNA (cDNA) per $100 \mathrm{~mm}$ dish using PolyFect transfection reagent (Qiagen) as per THE manufacturer's instructions. 
For SILAC experiments, MEFs were grown in either heavy SILAC DMEM $\left({ }^{13} \mathrm{C}_{6}\right.$-arginine, ${ }^{13} \mathrm{C}^{6}$-lysine; R6K6) or control SILAC DMEM $\left({ }^{12} \mathrm{C}_{6}\right.$-arginine, ${ }^{12} \mathrm{C}^{6}$ lysine; R0K0) (Dundee Cell Products, UK) supplemented with 10\% (v/v) dialysed calf serum, $100 \mathrm{U} \mathrm{ml}^{-1}$ penicillin, $100 \mu \mathrm{M}$ streptomycin, $4 \mu \mathrm{g} \mathrm{ml}^{-1}$ puromycin, 200 $\mathrm{mg}^{-1} \mathrm{~L}$-proline and $1 \mu \mathrm{M}$ D-biotin. Arginine can be metabolised from ${ }^{13} \mathrm{C}_{6}$ arginine to an isotope of the non-essential amino acid ${ }^{13} \mathrm{C}_{5}$-proline via the arginase pathway thus complicating data analysis ${ }^{70}$. As such, media were supplemented with L-proline $\left(200 \mathrm{mgl}^{-1}\right)$ to prevent arginine conversion. Furthermore, as overexpression of the HB-Ub-tag reduces the availability of cellular D-biotin ${ }^{21}$, growth medium was supplemented with D-biotin $(1 \mu \mathrm{M})$ to prevent saturation of in vivo biotinylation by excessive HB-Ub-tag expression. Plat-E retroviral packaging cells were maintained in DMEM supplemented with $10 \%$ (v/v) FBS, $100 \mathrm{U} \mathrm{ml}^{-1}$ penicillin, $100 \mu \mathrm{M}$ streptomycin, $1 \mu \mathrm{g} \mathrm{ml}^{-1}$ puromycin, $10 \mu \mathrm{g} \mathrm{ml}^{-1}$ blasticidin, and $1 \mathrm{mM}$ glutamine.

Constructs. Murine Grap2 (cat no. MR204666) and murine CIS (cat no. MR203328) in pCMV6-Entry were from Origene. Human SOCS3 CRISPR/Cas9 knockout (KO) and human SOCS3 HDR plasmids (cat. no. sc-400455) were from Santa Cruz Biotechnology. Expression constructs for Flag-tagged WT murine SOCS3 (hereafter termed pcDNA3/Flag-SOCS3), the truncation mutants $\Delta \mathrm{N} 20$, $\Delta \mathrm{N} 36, \Delta \mathrm{C} 40$, and $\Delta \mathrm{C} 84, \mathrm{R} 71 \mathrm{~K}$-mutated SOCS3, and the $\Delta \mathrm{PEST}$ mutant SOCS3 (generously provided by Dr Jeff Babon, Walter and Eliza Hall Institute of Medical Research, Australia) have all been described previously ${ }^{30,31,33,71}$. N-terminally GFP-tagged murine cavin-1 has been described previously ${ }^{32}$ while a cavin-1mCherry expression construct was generously provided by Dr Ben Nichols (MRC Laboratory of Molecular Biology, Cambridge, UK).

Full-length murine WT SOCS3, $\triangle$ SH2 SOCS3 (amino acids 46-142), and SOCS box domain-only (amino acids 177-225) GFP fusion proteins were generated by PCR amplification and sub-cloning in-frame with the GFP open reading frame in pEGFP-N1 (Clontech). The following primers were used to generate PCR products using pcDNA3/Flag-SOCS3 as the template prior to digestion with the indicated restriction enzymes for sub-cloning:-

Forward primers: WT (GAA GAA GAA TTC GCC ACC ATG GTC ACC CAC AGC AAG), SOCS box only (GAA GAA GAA TTC GCC ACC ATG GTA CTG AGC CGA CCT CTC), SH2 domain only (GAA GAA GAA TTC GCC ACC ATG TTC TAC TGG AGC GCC GTG). EcoRI sites for sub-cloning underlined, initiating Met codon in italics. Reverse primers: WT and SOCS box only (TT CTC GGG ATC CGC AAG TGG AGC ATC ATA CTG ATC CAG G). $\triangle$ SH2 SOCS3 (TTC TCG GGA TCC GCT TCC GTG GGT GGC AAA G). BamHI sites for subcloning underlined.

Myc-tagged WT murine cavin-1 and the truncation mutants N1 (amino acids 74-392), N2 (amino acids 168-392), N3 (amino acids 193-392), N4 (amino acids 250-392), C1 (amino acids 1-76), C2 (amino acids 1-167), C3 (amino acids 1-192), and C4 (amino acids 1-249) were generated by PCR amplification and subcloning in-frame with the C-terminal myc epitope (EQKLISEEDL) in pcDNA3.1/ mycHis A(-) (Invitrogen). The following primers were used to generate PCR products using $\mathrm{pEGFP}-\mathrm{C} 1 /$ cavin-1 as the template prior to digestion with the indicated restriction enzymes and sub-cloning:-

Forward primers: WT, C1, C3, C3 and C4 constructs (GGA GAA CCT CTA GAC GCC ACC ATG GAG GAT GTC ACG CTC), N1 (GGA GAA CCT CTA

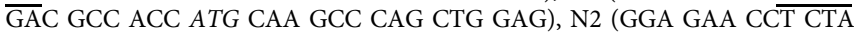

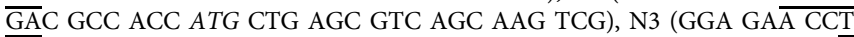
CTA GAC GCC ACC ATG CGG CCC GAG GAT GAC ACC), N4 (GGA GAA CCT CTA GAC GCC ACC ATG ACG CGT GAG AAC CTG GAG). XbaI sites for sub-cloning underlined, initiating Met codon in italics. Reverse primers: C1 (TTC TCG GAT CCA CTG GGC TTG GGT CAG CTG), C2 (TTC TCG GAT CCA TTT GGC CGG CAG CTT GAC), C3 (TTC TCG GAT CCA CTC GCC CTC GCC CAG CTC), C4 (TTC TCG GAT CCA GCG CAC CTT GGT CTT CTC). WT, N1, N2, N3, and N4 constructs (TTC TCG GAT CCA GTC GCT GTC GCT CTT GTC). BamHI sites for sub-cloning underlined.

A mutated Grap2(SOCS3-PEST) in which residues 129-163 encompassing the SOCS3 PEST sequence were inserted between amino acids 149-150 within the Grap2 ORF in pCMV6-Entry was synthesised by GeneArt ${ }^{\mathrm{TM}}$. All constructs were verified by sequencing to ensure the absence of additional unanticipated mutations.

Retroviral delivery of a $\mathbf{H i s}_{\mathbf{6}}+$ biotin $\mathrm{Ub}$ (HB-Ub) transgene. Using Lipofectamine2000 (Invitrogen), Plat-E retroviral packaging cells in $10 \mathrm{~cm}$ dishes at approximately $80 \%$ confluence were transfected with a HB-Ub-expressing plasmid kindly donated by Professor Peter Kaiser (University of California at Irvine, USA) 22. Following incubation in media without antibiotic selection, retroviruscontaining media were collected following two sequential incubation periods, one of $24 \mathrm{~h}$ at $37^{\circ} \mathrm{C}$ and a second of $24 \mathrm{~h}$ at $32^{\circ} \mathrm{C}$.

Retroviral-mediated generation of cell lines. MEFs in $10 \mathrm{~cm}$ dishes at approximately $40 \%$ confluence were transduced with $2 \mathrm{ml}$ of retrovirus containing media in a final volume of $4 \mathrm{ml}$ DMEM supplemented with $10 \%$ (v/v) FBS, $1 \mathrm{mM}$ glutamine, and $10 \mu \mathrm{g} \mathrm{ml}^{-1}$ polybrene. After $12 \mathrm{~h}$, the media were replaced with DMEM supplemented with $10 \%(\mathrm{v} / \mathrm{v}) \mathrm{FBS}, 1 \mathrm{mM}$ L-glutamine and $100 \mathrm{U} \mathrm{ml}^{-1}$ penicillin, $100 \mu \mathrm{M}$ streptomycin and $1 \mu \mathrm{g} \mathrm{ml}^{-1}$ puromycin to select for positive clones. Following dilution and re-plating, positive clones were expanded and HB-
Ub-expressing clones identified by immunoblotting whole-cell extracts with a polyHis antibody.

Tandem affinity purification. $\mathrm{SOCS}^{+/+}$and $\mathrm{SOCS}^{-/-}$MEFs were harvested in lysis buffer ( $8 \mathrm{M}$ urea, $300 \mathrm{mM} \mathrm{NaCl}, 50 \mathrm{mM} \mathrm{NaH}_{2} \mathrm{PO}_{4}, 0.5 \%$ (v/v) NP-40, pH 8.0) supplemented with $1 \mathrm{mM}$ phenylmethylsulfonyl fluoride (PMSF). Following sonication $(3 \times 10 \mathrm{~s}$ pulses, with a $10 \mathrm{~s}$ rest phase, at $40 \%$ amplitude), supernatants were isolated by centrifugation at $21,000 \times \mathrm{g}$ for $30 \mathrm{~min}$ at room temperature (RT) and equalised for protein content. Lysates from $\mathrm{SOCS}^{+/+}$and $\mathrm{SOCS3}^{-/-} \mathrm{MEFs}$ were mixed in a 1:1 ratio before incubation with $30 \mu \mathrm{l}$ of $50 \%(\mathrm{v} / \mathrm{v}) \mathrm{Ni}^{2+}-\mathrm{NTA}$ Sepharose beads per milligram of protein and rotated overnight at RT. Beads were isolated by centrifugation at $100 \times g$ for $1 \mathrm{~min}$ and then washed sequentially, once with 20 bead volumes of buffer A $\left(8 \mathrm{M}\right.$ urea, $300 \mathrm{mM} \mathrm{NaCl}, 50 \mathrm{mM} \mathrm{NaH} \mathrm{PO}_{4}$, $0.5 \%(\mathrm{v} / \mathrm{v}) \mathrm{NP}-40, \mathrm{pH} 8.0)$ supplemented with $1 \mathrm{mM}$ PMSF and $10 \mathrm{mM}$ imidazole and twice with 20 bead volumes of buffer B $(8 \mathrm{M}$ urea, $300 \mathrm{mM} \mathrm{NaCl}, 50 \mathrm{mM}$ $\mathrm{NaH}_{2} \mathrm{PO}_{4}, 0.5 \%(\mathrm{v} / \mathrm{v}) \mathrm{NP}-40, \mathrm{pH}$ 6.3) supplemented with $10 \mathrm{mM}$ imidazole and 1 $\mathrm{mM}$ PMSF. Beads were isolated by centrifugation at $100 \times \mathrm{g}$ for $1 \mathrm{~min}$ and bound proteins eluted twice with 5 bead volumes of elution buffer $(8 \mathrm{M}$ urea, $200 \mathrm{mM}$ $\mathrm{NaCl}, 50 \mathrm{mM} \mathrm{NaH} \mathrm{PO}_{4}, 2 \%$ (w/v) SDS, $10 \mathrm{mM}$ EDTA, $100 \mathrm{mM}$ Tris, $500 \mathrm{mM}$ imidazole, $\mathrm{pH}$ 8.0) supplemented with $1 \mathrm{mM}$ PMSF.

Eluate from the Ni affinity chromatography step was directly added to $10 \mu \mathrm{l}$ of $50 \%(\mathrm{v} / \mathrm{v})$ streptavidin-Sepharose beads per milligram of initial protein lysate and rotated overnight at RT. Beads isolated by centrifugation at $100 \times \mathrm{g}$ for $1 \mathrm{~min}$ at RT were washed sequentially, twice with 25 bead volumes of buffer C ( $8 \mathrm{M}$ urea, 200 $\mathrm{mM} \mathrm{NaCl}, 2 \%(\mathrm{w} / \mathrm{v})$ SDS, $100 \mathrm{mM}$ Tris, $\mathrm{pH} 8.0$ ) and twice with 25 bead volumes of buffer D ( $8 \mathrm{M}$ urea, $1.2 \mathrm{M} \mathrm{NaCl}, 0.2 \%$ (w/v) SDS, $100 \mathrm{mM}$ Tris, $10 \%$ (v/v) ethanol, $10 \%(\mathrm{v} / \mathrm{v})$ isopropanol, $\mathrm{pH} 8.0)$. Bound proteins were eluted with one bead volume of aqueous biotin $(50 \mathrm{mM})$ at $95^{\circ} \mathrm{C}$ for $5 \mathrm{~min}$. Following isolation by centrifugation at $100 \times g$ for $1 \mathrm{~min}$ at RT, eluate was concentrated using Amicon 10K Ultra-2 Centrifugal Filter Devices (Millipore) as per the manufacturer's instructions.

In-gel trypsin digestion. Sodium dodecyl sulphate-polyacrylamide gel electrophoresis (SDS-PAGE)-fractionated TAP eluate was stained with InstantBlue (Expedion) prior to manual sectioning into several manageable gel slices. Individual gel slices were washed sequentially with $500 \mu \mathrm{l}, 100 \mathrm{mM}$ ammonium bicarbonate and then $500 \mu \mathrm{l}, 50 \%(\mathrm{v} / \mathrm{v})$ acetonitrile/ammonium bicarbonate $(100 \mathrm{mM})$ for $30 \mathrm{~min}$ with shaking. The samples were reduced with the addition of $150 \mu \mathrm{l} 100$ $\mathrm{mM}$ ammonium bicarbonate and $10 \mu \mathrm{l} 45 \mathrm{mM}$ dithiothreitol for $30 \mathrm{~min}$ at $60^{\circ} \mathrm{C}$. Samples were cooled to RT before alkylation using $10 \mu \mathrm{l} 100 \mathrm{mM}$ iodoacetamide in the dark for $30 \mathrm{~min}$ at RT. Gel pieces were then washed in $500 \mu \mathrm{l} 50 \%(\mathrm{v} / \mathrm{v})$ acetonitrile/ammonium bicarbonate $(100 \mathrm{mM})$ for $1 \mathrm{~h}$ with shaking at RT. Following treatment with $50 \mu \mathrm{l}$ acetonitrile for $10 \mathrm{~min}$, the solvent was discarded and the gel pieces dried using a vacuum centrifuge for $1 \mathrm{~h}$. Gel slices were fully rehydrated in trypsin suspended in $1 \mathrm{ml} 25 \mathrm{mM}$ ammonium bicarbonate and incubated overnight at $37^{\circ} \mathrm{C}$ after which the supernatant was transferred to a fresh 96-well plate without disturbing the gel pieces. Residual digested protein was extracted by using $20 \mu \mathrm{l} 5 \%(\mathrm{v} / \mathrm{v})$ formic acid for $20 \mathrm{~min}$ at RT with shaking followed by the addition of $40 \mu \mathrm{l}$ acetonitrile for a further $20 \mathrm{~min}$ with shaking at RT. Pooled extracts were dried using a SpeedVac centrifugal evaporator before resuspension in $10 \mu \mathrm{ldH} 20$ prior to mass spectrometry.

Liquid chromatography and mass spectrometry. Samples were analysed on a Dionex Ultimate 3000 RSLS Nano flow system (Dionex). The samples $(5 \mu \mathrm{l})$ were loaded onto a Dionex $100 \mu \mathrm{m} \times 2 \mathrm{~cm} 5 \mu \mathrm{m} \mathrm{C18} \mathrm{Nano} \mathrm{trap} \mathrm{column} \mathrm{at} \mathrm{a} \mathrm{flow} \mathrm{rate} \mathrm{of}$ $5 \mu \mathrm{l} \mathrm{min}{ }^{-1}$ by the Ultimate $3000 \mathrm{RS}$ autosampler (Dionex). The composition of the loading solution was $0.1 \%$ formic acid and acetonitrile (98:2). Once loaded onto the column, the sample was then washed off into an Acclaim PepMap $75 \mu \mathrm{m} \times 15 \mathrm{~cm}$, $2 \mu \mathrm{m} 100 \AA \mathrm{C} 18 \mathrm{Nano}$ column at a flow rate of $0.3 \mu \mathrm{m} \mathrm{min}^{-1}$. The trap and nano flow column were maintained at $35^{\circ} \mathrm{C}$ in an UltiMate 3000 Rapid Separation LC system (Thermo Fisher). The samples were eluted with a gradient of solvent $\mathrm{A}$ : $0.1 \%$ formic acid (solvent A) versus acetonitrile (solvent B) starting at $1 \% \mathrm{~B}$ rising to $15 \%$ then to $45 \%$ B over 50 and then $90 \mathrm{~min}$. The column was washed using $90 \%$ $\mathrm{B}$ before being equilibrated prior to the next sample being loaded.

Column eluate was directed to a Proxeon Nano spray electrospray ionisation (ESI) source (Thermo Fisher) operating in positive ion mode and then into an Orbitrap Velos FTMS. The ionisation voltage was $2.5 \mathrm{kV}$ and the capillary temperature was $230^{\circ} \mathrm{C}$. The mass spectrometer was operated in tandem mass spectrometry (MS/MS) mode scanning from 300 to $2000 \mathrm{amu}$. The top 20 multiply charged ions were selected from each full scan for MS/MS analysis, the fragmentation method was CID at 35\% collision energy. The ions were selected for $\mathrm{MS}^{2}$ using a data-dependent method with a repeat count of $1 \mathrm{~s}$ and repeat and exclusion time of $15 \mathrm{~s}$. Precursor ions with a charge state of 1 were rejected. The resolution of ions in the first stage $\left(\mathrm{MS}^{1}\right)$ was 60,000 and 7500 for the second stage $\left(\mathrm{CID} \mathrm{MS}{ }^{2}\right.$ ). Data were acquired using Xcalibur v.2.1 (Thermo Fisher).

Analysis of LC-MS/MS. Post-LC-MS/MS analysis was performed using MaxQuant v.1.1.1.36 ${ }^{72}$ and searched with Andromeda search engine ${ }^{73}$ against the IPI mouse.v3.80 Fasta formatted database (release February 2011). Phosphorylation (S, $\mathrm{T}, \mathrm{Y}$ ), ubiquitination (GlyGly), and oxidation (Met) were set as variable 
modifications, whereas carbamidomethylation (Cys) was set as fixed modification. The peptides used for protein quantification were set to unique and razor and minimum ratio count set to 1 . Requantify was set to "TRUE" for deep searching of paired SILAC peaks. "Labelled amino acid filtering" was set to "FALSE" to improve analysis using R6K6 SILAC labelling. All other options were set to default.

CRISPR/Cas9 generation of SOCS3-null AS-M.5 EC lines. Using SuperFect transfection reagent (Qiagen), AS-M.5 cells in $6 \mathrm{~cm}$ dishes at approximately $80 \%$ confluence were co-transfected with human SOCS3 CRISPR/Cas9 KO and human SOCS3 HDR plasmids. Following dilution and re-plating, positive clones were isolated by selection in medium supplemented with puromycin $\left(2 \mu \mathrm{g} \mathrm{m}^{-1}\right)$. SOCS3-null clones were identified by immunoblotting whole-cell extracts with SOCS3 antibody following cellular treatment with Fsk $(50 \mu \mathrm{M}, 5 \mathrm{~h})$ to induce SOCS3 gene expression ${ }^{26,45}$

Antibodies. The following antibodies were obtained from the indicated suppliers: anti-Flag M2 antibody (Sigma F3165, 1 in 1000), anti-HA HA-7 antibody (Sigma H9658, 1 in 1000), PTRF/cavin-1 (Abcam ab48824, 1 in 1000), caveolin-1 (BD Biosciences 610059, 1 in 1000) and anti-phosphotyrosine monoclonal antibody 4G10 (Millipore 05-321, 1 in 1000), GAPDH (Abcam, ab8245, 1 in 20,000), antimyc 9E10 (ascites generated by ProSci, 1 in 2000), anti- $\alpha$-tubulin 12G10 (DSHB 12G10, 1 in 10,000), SOCS3 (M20; Santa Cruz sc-7009, 1 in 500), STAT3 (EPR787Y: Abcam ab68153, 1 in 1000), phospho-STAT3 (Tyr705) (3E2: Cell Signaling 9138L, 1 in 1000), phospho-STAT3 (Ser727) (6E4: Cell Signaling 9136, 1 in 1000), JAK1 (BD Transduction Laboratories 610232, 1 in 1000), JAK2 (D2E12: Cell Signaling 3230, 1 in 1000), and flotillin-1 (BD Transduction Laboratories 610821,1 in 500). Sheep polyclonal anti-GFP serum was generously provided by Professor Graeme Milligan (University of Glasgow, UK) and was used in a 1 in 2000 dilution.

Immunoblotting. Cell lysates were prepared as described previously ${ }^{74}$. Cells were washed twice with ice-cold phosphate-buffered saline (PBS) and lysed by scraping into lysis buffer (50 mM HEPES pH 7.4, $150 \mathrm{mM}$ sodium chloride, $1 \%$ (v/v) Triton $\mathrm{X}-100,0.5 \%(\mathrm{v} / \mathrm{v})$ sodium deoxycholate, $0.1 \%(\mathrm{w} / \mathrm{v}) \mathrm{SDS}, 10 \mathrm{mM}$ sodium fluoride, $5 \mathrm{mM}$ EDTA, $10 \mathrm{mM}$ sodium phosphate, $0.1 \mathrm{mM}$ PMSF, $10 \mu \mathrm{g} \mathrm{m}^{-1}$ benzamidine, $10 \mu \mathrm{g} \mathrm{ml}^{-1}$ soybean trypsin inhibitor, $2 \%$ (w/v) EDTA-free complete protease inhibitor cocktail (Sigma)). After $30 \mathrm{~min}$ on ice, lysates were vortexed and cleared by centrifugation. Equivalent amounts of protein, as determined by bicinchoninic acid protein assay, were resolved by SDS-PAGE, transferred to a nitrocellulose membrane, and analysed by immunoblotting as previously described ${ }^{26,74,75}$. Uncropped immunoblots used to generate Figs. $1 \mathrm{~b}$ and $5 \mathrm{~b}$ are shown in Supplementary Figure 7.

RNA analysis. Total RNA extraction from MEFs grown in $60 \mathrm{~mm}$ dishes was carried out using a miRNeasy Mini Kit (Qiagen) according to the manufacturer's instructions. The cDNA was generated from $1 \mu \mathrm{g}$ total RNA using $200 \mathrm{U}$ SuperScript ${ }^{\mathrm{TM}}$ II Reverse Transcriptase (Invitrogen) following the manufacturer's instructions with $100 \mathrm{ng}$ random hexamers, $2.5 \mathrm{mM}$ of each dNTP and $40 \mathrm{U}$ RNaseOUT (Invitrogen) in a final volume of $20 \mu \mathrm{l}$. Real-time quantitative PCRs were performed on a MX3000 system (Stratagene) using Power SYBR ${ }^{\circledast}$ Green PCR Master Mix (Applied Biosystems) in a final volume of $10 \mu \mathrm{l}$ containing $1 \mu \mathrm{l} \mathrm{cDNA}$, $0.5 \mathrm{mM}$ of each primer, and $1 \mathrm{x}$ Power SYBR ${ }^{\circledast}$ Green PCR Master Mix. The murine cavin-1 primers used were GCAAGGTCAGCGTCAAC (forward primer) and CCGGCAGCTTGACTTCA (reverse primer). GAPDH primers used for normalisation were GGCTGGCATTGCTCTCAA (forward primer) and

GCTGTAGCCGTATTCATTGTC (reverse primer). Primers were purchased from Dharmacon

Co-immunoprecipitation and peptide pull-down assays. For co-IP assays, either transfected cells or WT and SOCS3-null AS-M.5 cells were harvested in ice-cold PBS, pelleted by centrifugation at $1000 \times g$ for $5 \mathrm{~min}$ at $4{ }^{\circ} \mathrm{C}$, and lysed in co-IP buffer (50 mM HEPES, pH 7.4, $120 \mathrm{mM} \mathrm{NaCl}, 5 \mathrm{mM}$ EDTA, 10\% (v/v) glycerol, $1 \%(\mathrm{v} / \mathrm{v})$ Triton X-100, $5 \mathrm{mM} \mathrm{NaF}, 1 \mathrm{mM}$ sodium orthovanadate, $10 \mu \mathrm{g} \mathrm{ml}^{-1}$ benzamidine, $0.1 \mathrm{mM}$ PMSF, $10 \mu \mathrm{g} \mathrm{ml}^{-1}$ soybean trypsin inhibitor, $2 \%(\mathrm{w} / \mathrm{v})$ EDTA-free complete protease inhibitor cocktail). Following solubilisation by incubation for $1 \mathrm{~h}$ at $4{ }^{\circ} \mathrm{C}$ with rotation, lysates were centrifuged at $21,000 \times g$ for 15 min at $4{ }^{\circ} \mathrm{C}$ and the supernatant equalised for protein content and volume. Nonspecifically binding proteins were removed from soluble fractions by a $1 \mathrm{~h}$ preclearing step using $40 \mu \mathrm{l}$ of $50 \%(\mathrm{v} / \mathrm{v})$ slurry of protein G-Sepharose 4B FF beads (Sigma) re-suspended in $100 \mu \mathrm{l} 2 \%(\mathrm{w} / \mathrm{v})$ IgG-free bovine serum albumin (BSA). Following sedimentation of protein G-Sepharose beads by brief centrifugation, precleared lysates were incubated overnight at $4{ }^{\circ} \mathrm{C}$ with rotation with either $40 \mu \mathrm{l}$ fresh protein G-Sepharose beads pre-equilibrated with $2 \%(\mathrm{w} / \mathrm{v}) \mathrm{IgG}$-free BSA and anti-cavin-1 antibody or $40 \mu \mathrm{l}$ pre-conjugated anti-Flag M2-agarose beads (Sigma). Immune complexes were then isolated by brief centrifugation and washed three times with $1 \mathrm{ml}$ of co-IP buffer. Following removal of the final wash, protein complexes were eluted for analysis by SDS-PAGE by the addition of $40 \mu \mathrm{l}$ of electrophoresis sample buffer containing $12 \%(\mathrm{w} / \mathrm{v})$ SDS and incubation for $30 \mathrm{~min}$ at $65^{\circ} \mathrm{C}$ followed by a further $5 \mathrm{~min}$ at $95^{\circ} \mathrm{C}$.
For peptide pull-down assays, protein-equalised soluble extracts from transfected HEK293 cells were incubated with $100 \mathrm{nM} \mathrm{N}$-terminally biotinylated peptides (Severn Biotech, UK) and streptavidin-agarose prior to isolation of complexes by brief centrifugation and washing as described above. The peptides used had the following amino acid sequences: Tyr759 (Y), biotin-

TSSTVQYSTVVHSG; and PTyr759 (pY), biotin-TSSTVQpYSTVVHSG). Samples were then eluted for analysis by SDS-PAGE and immunoblotting as described above.

Peptide array overlays. Arrays were produced by automatic SPOT synthesis and synthesised on continuous cellulose membrane supports on Whatman 50 cellulose membranes using Fmoc-chemistry with the AutoSpot-Robot ASS 222 (Intavis Bioanalytical Instruments AG) as we have previously described ${ }^{76}$. Following blocking of non-specific protein binding sites by incubation in tris-buffered saline with Tween-20 (TBST; $50 \mathrm{mM}$ Tris $\mathrm{pH} 7.5,150 \mathrm{mM} \mathrm{NaCl}, 0.1 \%$ (v/v) Tween 20) containing $5 \%(\mathrm{w} / \mathrm{v}) \mathrm{BSA}$, membranes were overlaid with $10 \mu \mathrm{g} \mathrm{ml}^{-1}$ purified recombinant Trx-polyHis-tagged SOCS3 (Sino Biological Inc.) diluted in TBST-5\% (w/v) BSA. After washing in TBST, bound SOCS3 was detected by probing overlays with anti-SOCS3 antibody followed by IRDye-conjugated secondary antibody prior to visualisation using a LI-COR Odyssey Sa imaging system. As a negative control, identical arrays were identically treated in parallel minus recombinant SOCS3.

Subcellular fractionation. Confluent $10 \mathrm{~cm}$ dishes of WT, cavin-1-1-, and SOCS3 ${ }^{-1}$ MEFs were used to prepare subcellular fractions using a Subcellular Protein Fractionation Kit (Thermo Scientific) in accordance with the the manufacturer's instructions.

Confocal microscopy. For analysis of endogenous cavin-1 and transfected SOCS3GFP, WT, and cavin $-1^{-/-}$MEFs in $10 \mathrm{~cm}$ dishes were transiently transfected with or without SOCS3-GFP expression constructs. The following day, cells were split onto glass coverslips and left for a further $24 \mathrm{~h}$. Cells were then washed with PBS and fixed with $3 \%(\mathrm{w} / \mathrm{v})$ paraformaldehyde (PFA) in PBS for $25 \mathrm{~min}$. After washing with PBS and quenching residual PFA with $20 \mathrm{mM}$ glycine in PBS, cells were permeabilised with $0.1 \%(\mathrm{v} / \mathrm{v})$ Triton X-100 and non-specific binding sites blocked by a 30 min of incubation at RT in PBS containing 3\% (w/v) BSA and 10\% (v/v) donkey serum. Cells were then incubated with rabbit anti-cavin-1 antibody (Abcam ab48824, 1 in 100 dilution) for 90 min at RT. Cells were washed with PBS containing $0.1 \%(\mathrm{v} / \mathrm{v})$ Triton X-100, $1 \%(\mathrm{w} / \mathrm{v})$ BSA, and $10 \%(\mathrm{v} / \mathrm{v})$ donkey serum prior to incubation with Alexa Fluor 594-conjugated donkey anti-rabbit IgG (Life Technologies A21207, 1 in 200 dilution) for $1 \mathrm{~h}$ at RT. Finally, the cells were washed with PBS, mounted in ProLong ${ }^{\circ}$ Gold anti-fade reagent containing nuclear stain 4',6-diamidino-2-phenylindole (DAPI), and visualised using a LSM510 laser scanning confocal imaging system (Carl Zeiss). Images were analysed by Metamorph software to generate Pearson's correlation coefficients.

For experiments involving co-expression of SOCS3-GFP and cavin-1-mCherry in cavin-1-1- MEFs, cells at $80-90 \%$ confluence on $6 \mathrm{~cm}$ dishes were transfected with $1 \mu \mathrm{g}$ of each construct using PolyFect transfection reagent as per the manufacturer's instructions. The following day, cells were seeded into $\times 16 \mathrm{Lab}-\mathrm{Tek}$ chamber slides (Fisher Scientific) at a density of $5 \times 10^{4}$ cells per chamber and cultured for a further $24 \mathrm{~h}$. Cells were then washed twice with Hanks' balanced salt solution with $\mathrm{Ca}^{2+} / \mathrm{Mg}^{2+}$ and $0.2 \%$ (w/v) sucrose to preserve morphology before fixing by incubation with $4 \%$ (w/v) PFA at RT for $20 \mathrm{~min}$ in the dark. After removal of fixative and two washes with PBS, nuclei were stained with $10 \mu \mathrm{gl}^{-1}$ Hoechst 33342 (Life Technologies) prior to imaging using a VivaTome spinning disk confocal microscope (Carl Zeiss). Images were analysed using Fiji ImageJ and the Coloc 2 plugin.

Transmission electron microscopy. WT and SOCS3-null AS-M.5 cells were seeded at a density of $1 \times 10^{6}$ cells per $\mathrm{ml}$ into 6-well plates and onto Thermanox coverslips ( $13 \mathrm{~mm}$ diameter) for culturing to confluency. The cells were then fixed in $1.5 \%(\mathrm{w} / \mathrm{v})$ glutaraldehyde in $0.1 \mathrm{M}$ sodium cacodylate buffer at $4{ }^{\circ} \mathrm{C}$ for $1 \mathrm{~h}$. Following three washes in $0.1 \mathrm{M}$ sodium cacodylate buffer in $2 \%(\mathrm{w} / \mathrm{v})$ sucrose, the cells were incubated with $1 \%(\mathrm{w} / \mathrm{v})$ osmium tetroxide/0.1 M sodium cacodylate for $1 \mathrm{~h}$, washed three times in distilled water, and incubated in $0.5 \%(\mathrm{w} / \mathrm{v})$ uranyl acetate in the dark for $1 \mathrm{~h}$. Following two rinses in distilled water, cells were dehydrated in stepwise alcohol increments (30-100\% (v/v)) and incubated overnight in a 1:1 mix of propylene oxide/TAAB araldite Epon 812 resin. The propylene oxide was then allowed to evaporate to leave pure resin, which was changed twice before the sample was embedded in fresh resin which was allowed to polymerise at $60^{\circ} \mathrm{C}$ for $48 \mathrm{~h}$. Ultrathin sections were cut using a Leica Ultracut UCT and a Diatome diamond knife, contrast stained with aqueous $2 \%(\mathrm{w} / \mathrm{v})$ methanolic uranyl acetate and Reynolds lead citrate, and viewed using a LEO 912AB TEM (Carl Zeiss) at an accelerating voltage of $120 \mathrm{kV}$. TIF images were captured using an Olympus Soft Imaging System and image contrast modified using Adobe Photoshop CS.

Statistics. Statistical significance was assessed either by one-way analysis of variance or unpaired $t$-tests with an $\alpha$ probability of 0.05 . At least three separate experiments were used for analysis. 
Data availability. All the data that support the findings of this study are available from the corresponding author upon reasonable request.

Received: 24 July 2015 Accepted: 11 December 2017

Published online: 12 January 2018

\section{References}

1. O'Shea, J. J. et al. The JAK-STAT pathway: impact on human disease and therapeutic intervention. Annu. Rev. Med. 66, 311-328 (2015).

2. Eulenfeld, R. et al. Interleukin-6 signalling: more than Jaks and STATs. Eur. J. Cell Biol. 91, 486-495 (2012).

3. Quintás-Cardama, A., Kantarjian, H., Cortes, J. \& Verstovsek, S. Janus kinase inhibitors for the treatment of myeloproliferative neoplasias and beyond. Nat. Rev. Drug Discov. 10, 127-140 (2011).

4. O'Shea, J. J., Kontzias, A., Yamaoka, K., Tanaka, Y. \& Laurence, A. Janus kinase inhibitors in autoimmune diseases. Ann. Rheum. Dis. 72, iil11-iil15 (2013). Suppl 2.

5. Yu, H., Lee, H., Herrmann, A., Buettner, R. \& Jove, R. Revisiting STAT3 signalling in cancer: new and unexpected biological functions. Nat. Rev. Cancer 14, 736-746 (2014).

6. Linossi, E. M., Babon, J. J., Hilton, D. J. \& Nicholson, S. E. Suppression of cytokine signaling: the SOCS perspective. Cytokine Growth Factor Rev. 24, 241-248 (2013)

7. Babon, J. J., Varghese, L. N. \& Nicola, N. A. Inhibition of IL-6 family cytokines by SOCS3. Semin. Immunol. 26, 13-19 (2014).

8. Liu, E., Côté, J.-F. \& Vuori, K. Negative regulation of FAK signaling by SOCS proteins. EMBO J. 22, 5036-5046 (2003).

9. Niwa, Y. et al. Methylation silencing of SOCS-3 promotes cell growth and migration by enhancing JAK/STAT and FAK signalings in human hepatocellular carcinoma. Oncogene 24, 6406-6417 (2005).

10. Wölfler, A. et al. Site-specific ubiquitination determines lysosomal sorting and signal attenuation of the granulocyte colony-stimulating factor receptor. Traffic 10, 1168-1179 (2009).

11. Zhang, W.-N. et al. CUEDC2 (CUE domain-containing 2) and SOCS3 (suppressors of cytokine signaling 3 ) cooperate to negatively regulate Janus kinase 1/signal transducers and activators of transcription 3 signaling. J. Biol. Chem. 287, 382-392 (2012).

12. Palade, G. Fine structure of blood capillaries. J. Appl. Phys. 24, 1424 (1953).

13. Parton, R. G. \& del, Pozo, M. A. Caveolae as plasma membrane sensors, protectors and organizers. Nat. Rev. Mol. Cell Biol. 14, 98-112, (2013).

14. Liu, L. et al. Deletion of Cavin/PTRF causes global loss of caveolae, dyslipidemia, and glucose intolerance. Cell Metab. 8, 310-317 (2008).

15. Sehgal, P. B., Guo, G. G., Shah, M., Kumar, V. \& Patel, K. Cytokine signaling: STATS in plasma membrane rafts. J. Biol. Chem. 277, 12067-12074 (2002).

16. Podar, K. et al. Essential role of caveolae in interleukin-6- and insulin-like growth factor I-triggered Akt-1-mediated survival of multiple myeloma cells. J. Biol. Chem. 278, 5794-5801 (2003).

17. Elsasser, T. H. et al. Caveolae nitration of Janus kinase-2 at the $1007 \mathrm{Y}-1008 \mathrm{Y}$ site: coordinating inflammatory response and metabolic hormone readjustment within the somatotropic axis. Endocrinology 148, 3803-3813 (2007).

18. Lee, M. Y., Ryu, J. M., Lee, S. H., Park, J. H. \& Han, H. J. Lipid rafts play an important role for maintenance of embryonic stem cell self-renewal. J. Lipid Res. 51, 2082-2089 (2010).

19. Kershaw, N. J. et al. SOCS3 binds specific receptor-JAK complexes to control cytokine signaling by direct kinase inhibition. Nat. Struct. Mol. Biol. 20, 469-476 (2013).

20. Babon, J. J. et al. The SOCS box domain of SOCS3: structure and interaction with the elonginBC-cullin5 ubiquitin ligase. J. Mol. Biol. 381, 928-940 (2008).

21. Tagwerker, C. et al. A tandem affinity tag for two-step purification under fully denaturing conditions: application in ubiquitin profiling and protein complex identification combined with in vivo cross-linking. Mol. Cell Proteom. 5, 737-748 (2006).

22. Meierhofer, D., Wang, X., Huang, L. \& Kaiser, P. Quantitative analysis of global ubiquitination in HeLa cells by mass spectrometry. J. Proteome Res. 7, 4566-4576 (2008)

23. Hecht, D. \& Zick, Y. Selective inhibition of protein tyrosine phosphatase activities by $\mathrm{H} 2 \mathrm{O} 2$ and vanadate in vitro. Biochem. Biophys. Res. Commun. 188, 773-779 (1992).

24. Cox, J. \& Mann, M. MaxQuant enables high peptide identification rates, individualized p.p.b.-range mass accuracies and proteome-wide protein quantification. Nat. Biotechnol. 26, 1367-1372 (2008).

25. Ley, R. et al. Extracellular signal-regulated kinases $1 / 2$ are serum-stimulated "Bim(EL) kinases" that bind to the BH3-only protein Bim(EL) causing its phosphorylation and turnover. J. Biol. Chem. 279, 8837-8847 (2004).
26. Sands, W. A., Woolson, H. D., Milne, G. R., Rutherford, C. \& Palmer, T. M. Exchange protein activated by cyclic AMP (Epac)-mediated induction of suppressor of cytokine signaling 3 (SOCS-3) in vascular endothelial cells. Mol. Cell Biol. 26, 6333-6346 (2006).

27. Woolson, H. D., Thomson, V. S., Rutherford, C., Yarwood, S. J. \& Palmer, T. M. Selective inhibition of cytokine-activated extracellular signal-regulated kinase by cyclic AMP via Epac1-dependent induction of suppressor of cytokine signalling-3. Cell. Signal. 21, 1706-1715 (2009).

28. Krump-Konvalinkova, V. et al. Establishment and characterization of an angiosarcoma-derived cell line, AS-M. Endothelium 10, 319-328 (2009).

29. Fass, D. M., Butler, J. E. F. \& Goodman, R. H. Deacetylase activity is required for cAMP activation of a subset of CREB target genes. J. Biol. Chem. 278, 43014-43019 (2003).

30. Lehmann, U. et al. SHP2 and SOCS3 contribute to Tyr-759-dependent attenuation of interleukin-6 signaling through gp130. J. Biol. Chem. 278, 661-671 (2003).

31. Sasaki, A. et al. Cytokine-inducible SH2 protein-3 (CIS3/SOCS3) inhibits Janus tyrosine kinase by binding through the $\mathrm{N}$-terminal kinase inhibitory region as well as SH2 domain. Genes Cells 4, 339-351 (1999).

32. Hill, M. M. et al. PTRF-Cavin, a conserved cytoplasmic protein required for caveola formation and function. Cell 132, 113-124 (2008).

33. Babon, J. J. et al. Secondary structure assignment of mouse SOCS3 by NMR defines the domain boundaries and identifies an unstructured insertion in the SH2 domain. FEBS J. 272, 6120-6130 (2005).

34. Sandhu, K. S. \& Dash, D. Conformational flexibility may explain multiple cellular roles of PEST motifs. Proteins Struct. Funct. Bioinforma. 63, 727-732 (2006).

35. Sue, S.-C. \& Dyson, H. J. Interaction of the IkappaBalpha C-terminal PEST sequence with NF-kappaB: insights into the inhibition of NF-kappaB DNA binding by IkappaBalpha. J. Mol. Biol. 388, 824-838 (2009).

36. Babon, J. J. et al. The structure of SOCS3 reveals the basis of the extended $\mathrm{SH} 2$ domain function and identifies an unstructured insertion that regulates stability. Mol. Cell 22, 205-216 (2006).

37. Hansen, C. G. \& Nichols, B. J. Exploring the caves: cavins, caveolins and caveolae. Trends Cell Biol. 20, 177-186 (2010).

38. Hill, M. M. et al. Co-regulation of cell polarization and migration by caveolar proteins PTRF/Cavin-1 and caveolin-1. PLoS ONE 7, e43041 (2012).

39. Kaptein, A., Paillard, V. \& Saunders, M. Dominant negative stat3 mutant inhibits interleukin-6-induced Jak-STAT signal transduction. J. Biol. Chem. 271, 5961-5964 (1996).

40. Decker, T. \& Kovarik, P. Serine phosphorylation of STATs. Oncogene 19 , 2628-2637 (2000).

41. Heinrich, P. C. et al. Principles of interleukin (IL)-6-type cytokine signalling and its regulation. Biochem. J. 374, 1-20 (2003).

42. Croker, B. A. et al. SOCS3 negatively regulates IL-6 signaling in vivo. Nat. Immunol. 4, 540-545 (2003).

43. Lang, R. et al. SOCS3 regulates the plasticity of gp130 signaling. Nat. Immunol. 4, 546-550 (2003).

44. Brender, C. et al. Suppressor of cytokine signaling 3 regulates CD8 T-cell proliferation by inhibition of interleukins 6 and 27. Blood 110, 2528-2536 (2007).

45. Yarwood, S. J., Borland, G., Sands, W. A. \& Palmer, T. M. Identification of CCAAT/enhancer-binding proteins as exchange protein activated by cAMPactivated transcription factors that mediate the induction of the SOCS-3 gene. J. Biol. Chem. 283, 6843-6853 (2008).

46. Tagwerker, C. et al. A tandem affinity tag for two-step purification under fully denaturing conditions: application in ubiquitin profiling and protein complex identification combined with in vivo cross-linking. Mol. Cell. Proteom. 5, 737-748 (2006).

47. Garcia-Cardena, G. et al. Dissecting the interaction between nitric oxide synthase (NOS) and caveolin: functional significance of the nos caveolin binding domain in vivo. J. Biol. Chem. 272, 25437-25440 (1997).

48. Li, S., Couet, J. \& Lisanti, M. P. Src tyrosine kinases, galpha subunits, and h-ras share a common membrane-anchored scaffolding protein, caveolin. caveolin binding negatively regulates the auto-activation of src tyrosine kinases. J. Biol. Chem. 271, 29182-29190 (1996).

49. Buk, D. M. et al. Polarity and lipid raft association of the components of the ciliary neurotrophic factor receptor complex in Madin-Darby canine kidney cells. J. Cell Sci. 117, 2063-2075 (2004).

50. Yanagisawa, M., Nakamura, K. \& Taga, T. Roles of lipid rafts in integrindependent adhesion and gp130 signalling pathway in mouse embryonic neural precursor cells. Genes Cells 9, 801-809 (2004).

51. Yang, N., Huang, Y., Jiang, J. \& Frank, S. J. Caveolar and lipid raft localization of the growth hormone receptor and its signaling elements: impact on growth hormone signaling. J. Biol. Chem. 279, 20898-20905 (2004).

52. Park, D. S. et al. Caveolin-1-deficient mice show accelerated mammary gland development during pregnancy, premature lactation, and hyperactivation of the Jak-2/STAT5a signaling cascade. Mol. Biol. Cell 13, 3416-3430 (2002). 
53. Sotgia, F., Schubert, W., Pestell, R. G. \& Lisanti, M. P. Genetic ablation of caveolin-1 in mammary epithelial cells increases milk production and hyperactivates STAT5a signaling. Cancer Biol. Ther. 5, 292-297 (2006).

54. Mukhopadhyay, S. et al. Cytoplasmic provenance of STAT3 and PY-STAT3 in the endolysosomal compartments in pulmonary arterial endothelial and smooth muscle cells: implications in pulmonary arterial hypertension. Am. J. Physiol. Lung Cell. Mol. Physiol. 294, L449-L468 (2008).

55. Zhang, P.-J. et al. CUE domain containing 2 regulates degradation of progesterone receptor by ubiquitin-proteasome. EMBO J. 26, 1831-1842 (2007).

56. Schmitz, J., Weissenbach, M., Haan, S., Heinrich, P. C. \& Schaper, F. SOCS3 exerts its inhibitory function on interleukin-6 signal transduction through the SHP2 recruitment site of gp130. J. Biol. Chem. 275, 12848-12856 (2000).

57. Lin, Q. et al. The HECT E3 ubiquitin ligase NEDD4 interacts with and ubiquitinates SQSTM1 for inclusion body autophagy. J. Cell Sci. 130 , 3839-3850 (2017).

58. Morén, B. et al. EHD2 regulates caveolar dynamics via ATP-driven targeting and oligomerization. Mol. Biol. Cell 23, 1316-1329 (2012).

59. Bastiani, M. et al. MURC/Cavin-4 and cavin family members form tissuespecific caveolar complexes. J. Cell Biol. 185, 1259-1273 (2009).

60. Gambin, Y. et al. Single-molecule analysis reveals self assembly and nanoscale segregation of two distinct cavin subcomplexes on caveolae. Elife 3, e01434 (2014).

61. Ludwig, A. et al. Molecular composition and ultrastructure of the caveolar coat complex. PLoS Biol. 11, e1001640 (2013).

62. Subramaniam, P. S. \& Johnson, H. M. Lipid microdomains are required sites for the selective endocytosis and nuclear translocation of IFN-, Its receptor chain IFN- Receptor-1, and the phosphorylation and nuclear translocation of STAT1. J. Immunol. 169, 1959-1969 (2002).

63. Hayashi, Y. K. et al. Human PTRF mutations cause secondary deficiency of caveolins resulting in muscular dystrophy with generalized lipodystrophy. $J$. Clin. Invest. 119, 2623-2633 (2009).

64. Rajab, A. et al. Fatal cardiac arrhythmia and long-QT syndrome in a new form of congenital generalized lipodystrophy with muscle rippling (CGL4) due to PTRF-CAVIN mutations. PLoS Genet. 6, e1000874 (2010).

65. Dwianingsih, E. K. et al. Child with asymptomatic elevation of serum creatine kinase shows PTRF-CAVIN mutation matching with congenital generalized lipodystrophy type 4. Mol. Genet. Metab. 101, 233-237 (2010).

66. Ardissone, A. et al. Novel PTRF mutation in a child with mild myopathy and very mild congenital lipodystrophy. BMC Med. Genet. 14, 89 (2013).

67. Yajima, T. et al. Absence of SOCS3 in the cardiomyocyte increases mortality in a gp130-dependent manner accompanied by contractile dysfunction and ventricular arrhythmias. Circulation 124, 2690-2701 (2011).

68. Suessmuth, Y. et al. A new polycythaemia vera-associated SOCS3 SH2 mutant (SOCS3F136L) cannot regulate erythropoietin responses. Br. J. Haematol. 147, 450-458 (2009).

69. Kawaguchi, T. et al. Hepatitis $C$ virus down-regulates insulin receptor substrates 1 and 2 through up-regulation of suppressor of cytokine signaling 3 . Am. J. Pathol. 165, 1499-1508 (2004).

70. Bendall, S. C. et al. Prevention of amino acid conversion in SILAC experiments with embryonic stem cells. Mol. Cell Proteom. 7, 1587-1597 (2008).

71. Nicholson, S. E. et al. Mutational analyses of the SOCS proteins suggest a dual domain requirement but distinct mechanisms for inhibition of LIF and IL-6 signal transduction. EMBO J. 18, 375-385 (1999).

72. Cox, J. \& Mann, M. MaxQuant enables high peptide identification rates, individualized p.p.b.-range mass accuracies and proteome-wide protein quantification. Nat. Biotechnol. 26, 1367-1372 (2008).

73. Cox, J. et al. Andromeda: a peptide search engine integrated into the MaxQuant environment. J. Proteome Res. 10, 1794-1805 (2011).

74. Rutherford, C. et al. Regulation of cell survival by sphingosine-1-phosphate receptor S1P1 via reciprocal ERK-dependent suppression of Bim and PI-3kinase/protein kinase C-mediated upregulation of Mcl-1. Cell Death Dis. 4, e927 (2013).
75. Safhi, M. M. A., Rutherford, C., Ledent, C., Sands, W. A. \& Palmer, T. M. Priming of signal transducer and activator of transcription proteins for cytokine-triggered polyubiquitylation and degradation by the A $2 \mathrm{~A}$ adenosine receptor. Mol. Pharmacol. 77, 968-978 (2010).

76. Brown, K. M. et al. Phosphodiesterase-8A binds to and regulates Raf-1 kinase. Proc. Natl. Acad. Sci. USA 110, E1533-E1542 (2013).

\section{Acknowledgements}

The authors thank Drs Aki Yoshimura, Jeff Babon, and Ben Nichols for provision of expression constructs, Dr John Pediani for assistance with confocal imaging, and Mrs Margaret Mullin for assistance with TEM. This work was supported by project grants to T.M.P. from the Chief Scientist Office (ETM/226), British Heart Foundation (PG12/1/ 29276, PG 14/32/30812), and a National Health Service Greater Glasgow and Clyde Research Endowment Fund (2011REFCH08). P.F.P. was supported by the National Institutes of Health grant DK097708. J.J.L.W. was supported by a doctoral training studentship from the Biotechnology and Biological Sciences Research Council Doctoral Training Programme in Biochemistry and Molecular Biology at the University of Glasgow (BB/F016735/1). N.A. was supported by a Saudi Government PhD Scholarship. This work was also supported in part by equipment grants to T.M.P. from Diabetes UK (BDA 11/0004309) and Alzheimer's Research UK (ARUK-EG2016A-3).

\section{Author contributions}

J.J.L.W. designed and performed all the experiments except where indicated. Generation of the WT and SOCS3-null AS-M.5 cells and the data presented in Figs. 1b, c, 2b, c and $7 \mathrm{a}-\mathrm{c}$ and Supplementary Figs. 2, 5 and 6 were performed by N.A. Supplementary Figure 3 was generated by L.L. under the supervision of P.F.P. P.F.P. also provided technical advice on use of the cavin-1-1- MEFs. The proteomics experiments that resulted in the initial identification of cavin-1 were carried out under the supervision of W.M. and R.B. G.S.B. supervised the design and synthesis of the peptide array experiments. F.S. devised the peptide pull-down experiments and provided advice on use of several of the mutated SOCS3 constructs. T.M.P. conceived and designed the study, supervised the data analysis, and wrote the paper with input from all authors.

\section{Additional information}

Supplementary Information accompanies this paper at https://doi.org/10.1038/s41467017-02585-y.

Competing interests: The authors declare no competing financial interests.

Reprints and permission information is available online at http://npg.nature.com/ reprintsandpermissions/

Publisher's note: Springer Nature remains neutral with regard to jurisdictional claims in published maps and institutional affiliations.

Open Access This article is licensed under a Creative Commons Attribution 4.0 International License, which permits use, sharing, adaptation, distribution and reproduction in any medium or format, as long as you give appropriate credit to the original author(s) and the source, provide a link to the Creative Commons license, and indicate if changes were made. The images or other third party material in this article are included in the article's Creative Commons license, unless indicated otherwise in a credit line to the material. If material is not included in the article's Creative Commons license and your intended use is not permitted by statutory regulation or exceeds the permitted use, you will need to obtain permission directly from the copyright holder. To view a copy of this license, visit http://creativecommons.org/ licenses/by/4.0/.

(C) The Author(s) 2018 